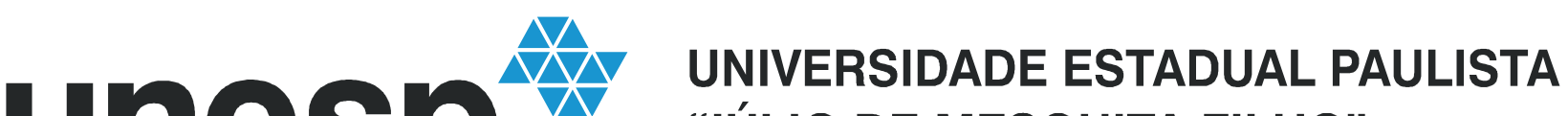 "JÚLIO DE MESQUITA FILHO"
}

Programa de Pós Graduação em Fisioterapia

“ASSOCIAÇÃO ENTRE ATIVIDADE FÍSICA E COMORBIDADES DE PACIENTES HIPERTENSOS EM TRATAMENTO

AMBULATORIAL"
Denise Rodrigues Bueno

Presidente Prudente - SP

2010 
Denise Rodrigues Bueno

\section{ASSOCIAÇÃO ENTRE ATIVIDADE FÍSICA E COMORBIDADES DE PACIENTES HIPERTENSOS EM TRATAMENTO AMBULATORIAL}

Dissertação apresentada à Faculdade de Ciências e Tecnologia - Universidade Estadual Paulista, campus de Presidente Prudente, para obtenção do título de Mestre em Fisioterapia.

Orientador: Prof. Dr. Henrique Luiz Monteiro

Presidente Prudente 


\section{Bueno, Denise Rodrigues.}

B941a Associação entre nível de atividade física e ocorrência de comorbidades de pacientes hipertensos em tratamento ambulatorial / Denise Rodrigues Bueno. - Presidente Prudente: [s.n], 2010 $\mathrm{xi}, 52 \mathrm{f}$.

Dissertação (mestrado) - Universidade Estadual Paulista, Faculdade de Ciências e Tecnologia

Orientador: Henrique Luiz Monteiro

Banca: Luiz Carlos Marques Vanderlei, Alberto de Vitta Inclui bibliografia

1. Atividade física. 2. Comorbidades. 3. Hipertensão Arterial. I. Autor. II. Universidade Estadual Paulista. Faculdade de Ciências e Tecnologia. III. Título.

CDD(18.ed.) 796

Ficha catalográfica elaborada pela Seção Técnica de Aquisição e Tratamento da Informação - Serviço Técnico de Biblioteca e Documentação - UNESP, Câmpus de Presidente Prudente. 


\section{AGRADECIMENTOS}

Agradeço a Deus pela força e fé que nunca faltaram e que ajudaram a chegar ao final.

À minha família pelo total apoio que se mostraram presentes sempre. Amo vocês.

Especialmente à minha mãe, meu irmão e meu pai, pelo amor e carinho. Caminharam juntos comigo o tempo todo, e não me deixaram faltar nada, nem a certeza que todas as dificuldades tinham seu propósito!

Aos fiéis amigos de Presidente Prudente, que mesmo distantes não deixaram de ser importantes e prover palavras de otimismo e carinho.

Aos novos amigos que fiz em Bauru, pois ajudaram da forma que puderam, e em tão pouco tempo se tornaram especiais.

Ao meu orientador, Prof. Dr. Henrique Luiz Monteiro, pela paciência, pelo tempo perdido com incansáveis correções dos manuscritos, e pelos inúmeros ensinamentos neste período

de convivência.

Ao Prof. Dr. Ismael F. Freitas Jr, pois sem ele nada teria acontecido, à professora e amiga Ms. Camila Buonani, pela amizade e ajuda direta na dissertação, por fim, ao CELAPAM, por ceder parte dos materiais necessários para a realização desta pesquisa.

Aos responsáveis pelos núcleos de saúde que ajudaram nas coletas, cederam com materiais, espaço e os pacientes.

Às professoras e amigas Ms. Jamile e Clara que me acolheram no momento crucial de toda a etapa e se mostraram companheiras, de amizade inestimável. Sentirei saudades! 
"O que você ganha, ao atingir seu objetivo, não é tão importante quanto o que você se torna ao atingi-lo."

(Zig Ziglar) 


\section{SUMÁRIO}

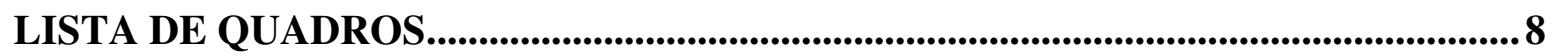

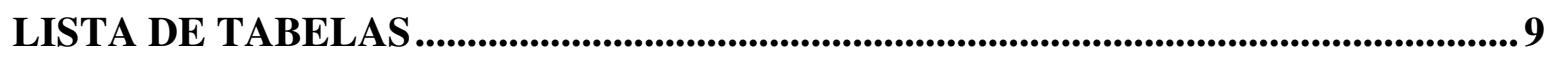

LISTA DE ILUSTRAÇÕES .............................................................................10

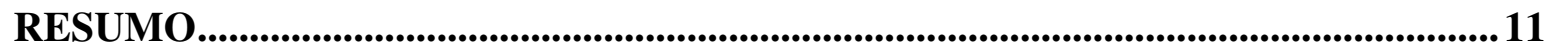

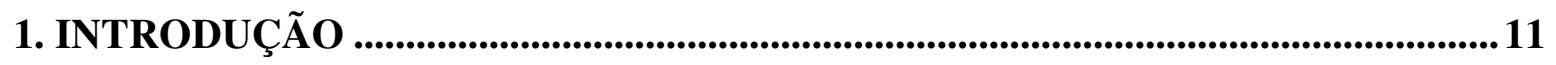

2. OBJETIVOS …………................................................................................................................ 14

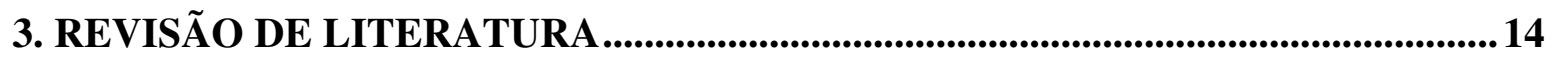

3.1 Hipertensão Arterial Sistêmica......................................................................... 14

3.2 Tipos de Atividade Física....................................................................................... 18

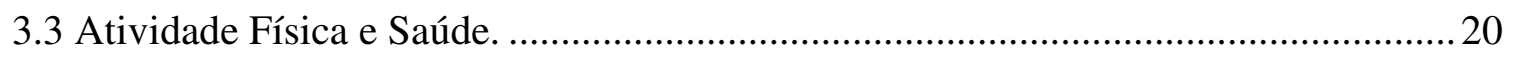

3.4 Nível de Atividade Física e Hipertensão Arterial........................................................22

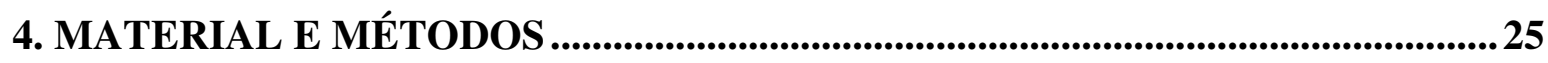

4.1 Natureza da pesquisa, casuística e local de estudo .....................................................25

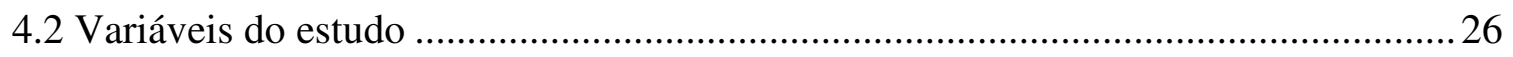

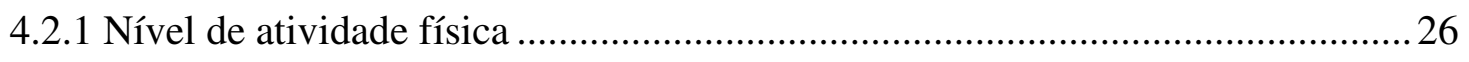

4.2.2 Distúrbios metabólicos, cardiovasculares e osteomusculares. .............................28

4.2.3 Avaliação do paciente.......................................................................................29

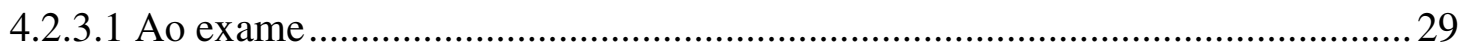

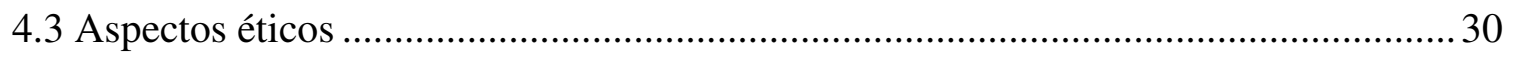

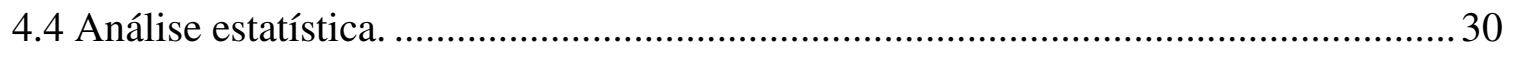

5. RESULTADOS …................................................................................................................... 30

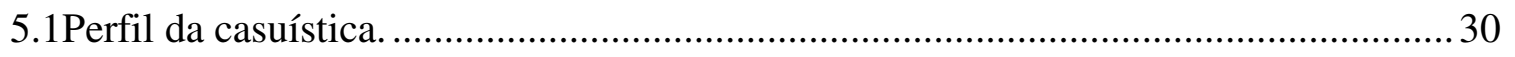

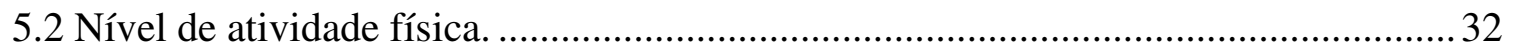

5.3 Presença de doenças. ............................................................................................. 35

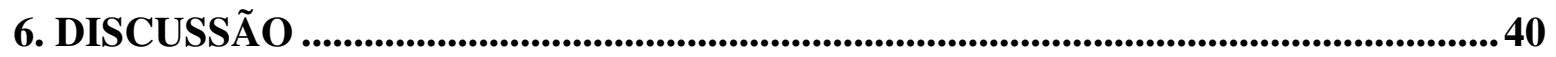




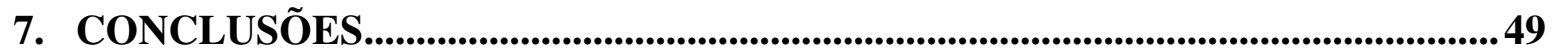

8. REFERÊNCIAS ......................................................................................................5

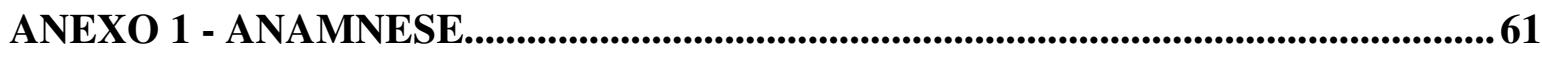

ANEXO 2 - INQUÉRITO DE MORBIDADES ..............................................................62

ANEXO 3 - NÍVEL DE ATIVIDADE FÍSICA ................................................................63 


\section{LISTA DE QUADROS}

Quadro 1. Valores de Pressão Arterial Sistólica (PAS) e Diastólica (PAD), e classificação

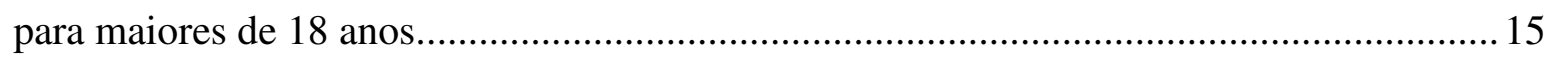

Quadro 2. Classificação internacional de adultos abaixo do peso, com sobrepeso e obesos,

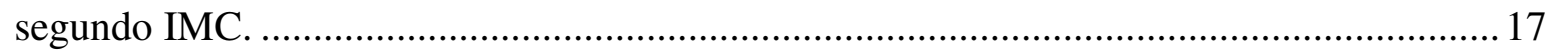

Quadro 3. Número de pacientes em cada grupo de acordo com o nível de atividade física

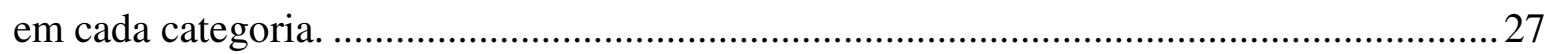

Quadro 4. Classificação dos dados sócio-demográficos segundo fatores de risco e de

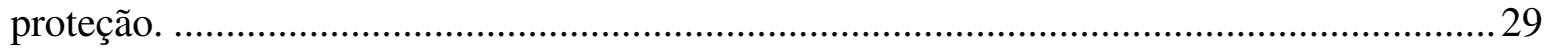




\section{LISTA DE TABELAS}

Tabela 1. Valores descritivos de média e desvio padrão para variáveis antropométricas, pressão arterial, e escores das categorias de atividade física, para homens e mulheres........31

Tabela 2. Distribuição da freqüência dos pacientes estudados, segundo caracterização e sexo....... 32

Tabela 3. Valores de média e desvio padrão dos escores de atividade física divididos por categoria e níveis de atividade física. 33

Tabela 4. Média e desvio padrão dos dados antropométricos, pressão arterial e idade, dividido por categoria da atividade física. 34

Tabela 5. Regressão linear ( $\beta$ ) entre variáveis antropométricas, idade e pressão arterial, com escore de atividade física. 35

Tabela 6. Distribuição de acordo com fatores de risco em relação presença de doenças. ...36

Tabela 7. Distribuição da casuística de acordo com nível de atividade física entre os grupos de doenças (CID).

Tabela 8. Distribuição da casuística segundo número de fatores de risco em relação à ausência de doenças, nível e categoria de atividade física. 38 


\section{LISTA DE ILUSTRAÇÕES}

Ilustração 1. Regressão linear entre pressão arterial sistólica (PAS) e categorias de atividade física, com respectivos valores de significância (p) para a correlação entre as variáveis. *=

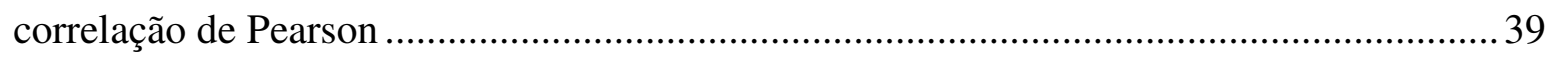

Ilustração 2. Regressão linear entre valores de pressão arterial diastólica (PAD) e escores de

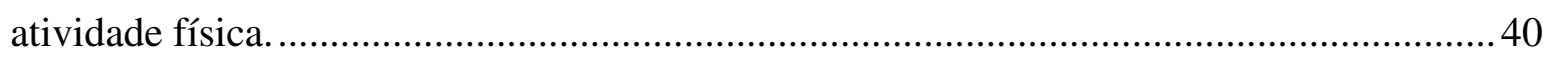




\section{RESUMO}

A presente pesquisa destinou-se a investigar as associações entre o nível de atividade física (NAF) de diferentes categorias, de pacientes hipertensos em tratamento farmacológico, com variáveis antropométricas e ocorrência de comorbidades. A pesquisa foi realizada na cidade de Bauru, junto aos Núcleos de Saúde "Otávio Rasi" e "Vila Cardia". Foram avaliados 192 pacientes, com média de idade de $63 \pm 11$ anos. As medidas de massa corporal total, estatura (cálculo do $\mathrm{IMC}=\mathrm{Kg} / \mathrm{m}^{2}$ ) e circunferência de cintura $(\mathrm{CC})$ foram empregadas como indicadores de estado nutricional e risco coronariano. A estimativa do NAF foi

realizada aplicando-se questionário de Baecke et al. ${ }^{(90)}$, e, após computados os escores para cada categoria de atividade física (ocupacional=AFO; tempo livre=AFTL; locomoção=AFL). Utilizando-se o valor médio do escore como ponto de corte para divisão dos grupos (ativo e sedentário) em cada categoria de atividade física. As comorbidades associadas foram identificadas aplicando-se um inquérito fechado de morbidade referida. Para a análise estatística aplicou-se o teste $T$ de Student para dados não pareados nas comparações entre ativos e sedentários; o teste Qui-quadrado para comparação de proporções, e o teste de correlação de Pearson foi empregado para análise entre variáveis contínuas. Resultados mostram a prevalência de indivíduos da cor branca $(61,5 \%)$, baixa escolaridade (ginásio incompleto: 50,0\%) e a AFTL como categoria de atividade física com escore mais elevado $(3,48 \pm 1,50)$. Diferenças entre ativos e sedentários em relação à antropometria e PA de repouso foram observadas apenas para a AFTL e AF Total. Correlações negativas significantes foram observadas entre as categorias AFO e AF Total com a idade ( $\mathrm{p}<0,05)$; AFL e AF Total com PAS ( $\mathrm{p}=0,02$ e $\mathrm{p}=0,008$, respectivamente). As atividades AFTL e AF total influenciam presença de doenças endócrino-metabólicas; não houve relação entre categorias de atividade física com outros tipos de doenças. Conclusão: A ocorrência de comorbidades não parece ser determinada pelo nível de atividade física nesta amostra, com exceção para as categorias AFTL e AFL e doenças metabólicas. Variáveis antropométricas IMC, CC e peso corporal total também parecem não ser influenciadas pela categoria de atividade física, comparando-se pacientes sedentários e ativos em cada uma delas; a pressão arterial sofreu influencia das categorias de AFTL e AF Total, sempre com menores valores observados entre os pacientes ativos. 


\begin{abstract}
This study aimed to investigate the associations of physical activity in different categories of hypertensive patients treated with drug therapy, anthropometric variables and comorbidities. The survey was conducted in Bauru city, in collaboration with the Centers for Health "Otavio Rasi" and "Vila Cardia" that are served by project linked to Unesp. We evaluated 192 patients with a mean age of 63,1 $\pm 11,05$ years. Height and waist circumference were measured for total body mass, and used as indicators of nutritional status and coronary risk. The estimated level of physical activity was performed by applying the Baecke et al., ${ }^{(90)}$ questionnaire and, after counting the scores for categories of physical activity $(\mathrm{OC}=$ occupational; $\mathrm{LTPA}=$ Leisure-time; $=\mathrm{LPA}=$ locomotion $)$ patients were divided into sedentary (SED) and physically active (PA) in each class studied, using the mean score as the cutoff point to divide the groups. Comorbidities were identified applying the morbidity inquiry. Both surveys were administered through interviews. Statistical analysis was made by Student t-test for unpaired comparisons between active and sedentary; chi-square test was used to compare proportions and to assess the association between the independent variables, one-way ANOVA was used in comparisons between two or more variables. Results show the prevalence of individuals of white, low education and AFLT with the highest score. Differences between active and sedentary in relation to anthropometry and resting BP were observed for the LTPA and Total PA. Significant correlations were observed between OC and Total PA in aged, and LPA, and, Total PA with SBP. Conclusions: The occurrence of comorbidities not seem to be determined by the level of physical activity in this sample, except for categories LTPA and LPA and metabolic diseases, anthropometric variables BMI, WHR and total body weight also appear to be influenced by the category of physical activity, compared is active and sedentary patients in each, the pressure has influenced the categories of LTPA and Total PA, always with lower values observed.
\end{abstract}




\section{INTRODUÇÃO}

A hipertensão arterial sistêmica (HAS) é uma doença considerada fator de risco para complicações cardiovasculares. É responsável por elevado número de hospitalizações e mortes em todo o mundo, especialmente em países em desenvolvimento, como o Brasil ${ }^{(1)}$. Relações entre a doença e aspectos relacionados ao sedentarismo, hábitos alimentares inadequados e incidência de morbi-mortalidade associada têm sido descritas na literatura nas últimas décadas ${ }^{(2-4)}$.

Pesquisas destinadas a identificar pontos de corte para o nível de atividade física necessário para manter saúde cardiovascular e, adicionalmente promover proteção contra a HAS, apresentam erros e diferenças metodológicas geradoras de conflitos no que diz respeito às afirmações sobre a quantidade de atividade física e os benefícios do esquema de

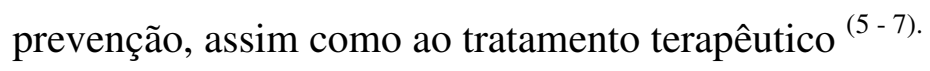

Sabe-se, entretanto, que a prática regular de exercícios físicos (EF), particularmente o aeróbio contínuo, e a consequente manutenção da aptidão do sistema cardiorrespiratório, podem resultar em redução dos valores de pressão arterial no organismo ${ }^{(8-10)}$. No presente trabalho assume-se o conceito de EF como subcategoria de atividade física (AF) que resulta em elevado gasto energético. A literatura preconiza que acumular tarefas como caminhadas com duração maior que trinta minutos, subir escadas, entre outras ${ }^{(1)}$, tanto de natureza ocupacional como de tempo livre, também podem resultar em proteção cardiovascular ${ }^{(11,}$ 12).

Os profissionais que prescrevem EF direcionados à saúde, por vezes adotam a recomendação atual que preconiza pelo menos 30 minutos de atividades físicas diárias (no mínimo 150 minutos por semana) como forma de prevenir doenças metabólicas e 
cardiovasculares, bem como manter a capacidade funcional ${ }^{(13)}$. No entanto, indivíduos que apresentam comprometimentos motores dificilmente obtêm os benefícios das atividades na quantidade preconizada, pois devido aos agravos osteoarticulares esta tarefa se torna mais difícil. Desse modo, é preciso buscar alternativas de incorporar as atividades físicas à vida dessas pessoas, de tal forma que, possam controlar a pressão arterial, sem efeitos colaterais sobre o sistema músculo-esquelético.

Pesquisas que buscaram identificar se atividades de lazer ou ocupacionais têm impacto sobre parâmetros hemodinâmicos e metabólicos, mostraram resultados distintos (11, ${ }^{14,15)}$, ou pouco expressivos ${ }^{(16)}$. Fagard ${ }^{(17)}$ afirma que os achados ainda são conflitantes com relação à menor incidência de HAS com maiores níveis de AF. Estudos de revisão ${ }^{(14)}$ constatam a necessidade de mais investigações acerca das atividades ocupacionais e de vida diária que resultem em menores valores pressóricos e proteção ao organismo.

Entre os desafios a serem superados, quantificar o nível de AF da população tem sido desafio de estudos nacionais ${ }^{(18,19)}$ e internacionais ${ }^{(20,21)}$. Doenças crônicas como a obesidade ${ }^{(22-24)}$ hipertensão ${ }^{(5,25)}$, diabetes ${ }^{(26)}$, dislipidemias ${ }^{(27,28)}$, entre outras ${ }^{(29,30)}$, de modo geral, são inversamente associadas à adesão à prática de atividades físicas ${ }^{(13)}$. Contudo, a inconsistência nos resultados de pesquisas que buscaram identificar a relação entre AF e o impacto sobre a HAS ${ }^{(31,32)}$ indicam lacunas na literatura e a necessidade de melhor controle das variáveis intervenientes, com o intuito de identificar a quantidade de atividade a ser incorporada com o objetivo de prevenção e tratamento da doença, principalmente em relação à população brasileira.

A dificuldade de se aprofundar as investigações sobre o problema é complexo, também pelo fato dos estudos longitudinais serem raros e de alto custo ${ }^{(19)}$. Sobre este assunto, pouco se conhece a respeito da população brasileira acerca da relação exercício 
físico e saúde. De modo geral, as orientações realizadas por profissionais da área são oriundas de resultados de pesquisa provenientes de países desenvolvidos, como as recomendações da Organização Mundial de Saúde (OMS), American College of Sports Medicine (ACSM), entre outras.

As diretrizes do ACSM ${ }^{(13)}$ para a saúde pública indicam os exercícios de intensidade moderada e vigorosa como melhores indutores de adaptações fisiológicas que oferecem proteção ao surgimento de doenças crônicas. No entanto é preciso considerar o efeito das atividades do cotidiano como coadjuvantes no processo saúde-doença. Estudos brasileiros direcionados para a investigação das causas da hipertensão, sua relação com os exercícios físicos e estilo de vida, são importantes para fundamentar o trabalho de profissionais da área, para o delineamento de programas de intervenção e, principalmente, a identificação das formas de prevenção.

Adicionalmente, é importante identificar as consequências da associação da HAS com outras doenças crônicas bem como o quanto à atividade física pode promover benefícios, diminuir custos com tratamento farmacológico ou prevenir a incidência de outras morbidades associadas ao estilo de vida e HAS.

Por este motivo é que se propõe a presente pesquisa a investigar associação entre nível de atividade física em suas diferentes categorias (atividade física ocupacional, de tempo livre e de locomoção), com variáveis antropométricas consideradas indicadoras de saúde, e ocorrência de comorbidades em sujeitos hipertensos. 


\section{OBJETIVOS}

Investigar as associações entre o nível de atividade física de diferentes categorias, de pacientes hipertensos em tratamento farmacológico, com variáveis antropométricas e ocorrência de comorbidades.

De modo mais específico, verificar:

$\checkmark$ Presença de outras doenças crônicas e sua associação com o nível de atividade física;

$\checkmark$ Associação entre as categorias de atividade física com presença de doenças crônicas e controle da HAS.

\section{REVISÃO DE LITERATURA}

\subsection{Hipertensão Arterial Sistêmica.}

A HAS é uma doença caracterizada por elevados níveis tensionais sanguíneos sobre as paredes arteriais; de caráter multifatorial, dentre os quais estão fatores genéticos, ambientais, neuro-humorais, vasculares, renais e cardiogênicos. Segundo a V Diretriz Brasileira de Hipertensão Arterial ${ }^{(1)}$, é considerado hipertenso o indivíduo que apresenta, em repouso, pressão arterial sistólica $(P A S) \geq 140 \mathrm{mmHg}$, e diastólica $(\mathrm{PAD}) \geq 90 \mathrm{mmHg}$ (Quadro 1). 
Quadro 1: Valores de Pressão Arterial Sistólica (PAS) e Diastólica (PAD): classificação para maiores de 18 anos.

\begin{tabular}{|lcc|}
\hline Classificação & PAS $-\mathbf{\text { mmHg}}$ & PAD $-\mathbf{\text { mmHg}}$ \\
\hline Ótima & $<120$ & $<80$ \\
Normal & $<130$ & $<85$ \\
Limítrofe & $130-139$ & $85-89$ \\
Hipertensão (estágio 01) & $140-159$ & $90-99$ \\
Hipertensão (estágio 02) & $160-179$ & $100-109$ \\
Hipertensão (estágio 03) & $>180$ & $>110$ \\
Hipertensão sistólica (isolada) & $>140$ & $>90$ \\
\hline
\end{tabular}

FONTE: Adaptado de V DBHA (2006)

Estudos de prevalência no Brasil são escassos. Passos et al., (33) em 2006, identificaram treze trabalhos com metodologia considerada adequada (que permita extrapolações e inferências corretas). Na presente comunicação, a prevalência média na população brasileira adulta encontra-se em torno de $20 \%$, e demonstra tendência de aumento dos casos em relação direta com a progressão da idade. De acordo com o Guideline Latino Americano de Hipertensão ${ }^{(34)}$ publicado em 2009, a prevalência estimada no Brasil varia de $25 \%$ a $35 \%$ na população adulta.

A HAS é fortemente associada com fatores de risco relacionados ao estilo de vida. Consumo excessivo de álcool, sedentarismo e alimentação inadequada (alta ingestão calórica e de sódio), são exemplos tipificados como risco para o aumento da pressão arterial. A esse propósito, Xin et al., ${ }^{(35)}$, apontam que a diminuição do consumo de álcool foi correspondente à queda da PAS $(-3,31 \mathrm{mmHg})$ e da PAD $(-2,04 \mathrm{mmHg})$ com o efeito dose-dependente. Segundo Wehlton et al., ${ }^{(36)}$ a combinação do consumo moderado de álcool/ sódio somado ao aumento do nível de atividade física e ingestão de frutas e verduras, é o melhor método de prevenção da HAS na população geral e grupos de risco. 
Appel et al., ${ }^{(37)}$ conduziram o PREMIER Clinical Trial 2003 e no referido trabalho analisaram os resultados sobre o comportamento da PA de indivíduos expostos a diferentes intervenções. O grupo sob restrição da ingestão de álcool e sal, controle do peso corporal e aumento da atividade física diária foi o que registrou a menor ocorrência de HAS quando comparado ao que apenas recebeu informações sobre os fatores de risco para o desenvolvimento da doença. Estas investigações evidenciam a interação entre diferentes fatores diversos na etiologia da doença e apontam a importância de amplas mudanças do estilo de vida para o efetivo controle dos valores pressóricos.

O cuidado com os hábitos de vida revela-se importante para a prevenção da HAS, evitando alterações nas estruturas do aparelho circulatório, tais como o espessamento da parede vascular, bem como outras patologias como a insuficiência renal, eventos cardiovasculares e elevada taxa de morbi-mortalidade em homens e mulheres de todo o mundo ${ }^{(38,39)}$. Os estudos epidemiológicos de Steptoe e McMunn ${ }^{(40)}$ e de Vasan et al., ${ }^{(41)}$ demonstram a relação entre PAS/PAD e agravos cardiovasculares em diferentes grupos de indivíduos com características distintas. No trabalho de Greenberg ${ }^{(42)}$, a PAS aumentada mostrou ser forte indicadora de Doenças Cardiovasculares (DCV) em homens saudáveis diagnosticados como pré-hipertensos. O aumento de $10 \mathrm{mmHg}$ na PA representou o incremento do risco relativo para $\mathrm{DCV}(\mathrm{RR}=1,43$ para $\mathrm{PAD} ; 2,11$ para $\mathrm{PAS})$. Corroborando com esses dados, Brown, Giles e Greenlund ${ }^{(43)}$ encontraram aumento significante $(p<0,05)$ no risco relativo de morte súbita entre homens $(R R=1,19)$ e mulheres $(\mathrm{RR}=1,15)$, associado com a PAS.

Demonstrando a mesma tendência, o risco relativo de hipertensos desenvolverem DCV em pesquisa publicada por Weycker et al., ${ }^{(44)}$ foi de 2,07 quando os doentes eram também diabéticos. Associando as duas patologias ao IMC e à hiperlipidemia o risco 
aumenta para 2,80. O estudo de Framigham evidencia este quadro preocupante, mostrando a relação entre HAS e fatores de risco, no qual foi encontrado menos de $20 \%$ de hipertensos com apenas um único fator de risco para $\mathrm{DCV}^{(45)}$.

Esses dados sugerem o controle da PA com impacto direto sobre a longevidade e prevenção e eventos cardiovasculares diversos, o que pode em contrapartida, reduzir custos com tratamento ambulatorial, principalmente relacionado às despesas com programas de reabilitação. O levantamento realizado em grupo de reabilitação cardiovascular na cidade de Presidente Prudente, SP, frequentado predominantemente por idosos ${ }^{(46)}$, encontrou HAS como doença de maior ocorrência (35\%) entre os pacientes atendidos.

A identificação de fatores de risco e investigação das causas da HAS tem utilizado parâmetros antropométricos como uma das ferramentas empregadas. Medidas que permitem o uso de taxas que estimem o risco cardiovascular como o índice de massa corporal (IMC - Quadro 2) e a circunferência de cintura (CC) são amplamente utilizadas. Estas mensurações também constituem indicadores de outras doenças como o diabetes mellitus, afecções cardiovasculares e mortalidade em indivíduos de diferentes faixas etárias (47). Há estudos que constataram maior prevalência de HAS entre pessoas com valores elevados de $\operatorname{IMC}^{(2,41,48)}$, de circunferência de cintura ${ }^{(49-51)}$ e peso corporal ${ }^{(52)}$.

Quadro 2. Classificação internacional de adultos abaixo do peso, com sobrepeso e obesos, segundo IMC.

\begin{tabular}{|lc|}
\hline Classificação & $\mathrm{IMC}\left(\mathrm{Kg} / \mathrm{m}^{2}\right)$ \\
\hline Abaixo do peso & $<18,50$ \\
Normal & $18,50-24,99$ \\
Sobrepeso & $25,0-29,9$ \\
Obesidade & $\geq 30$ \\
\multicolumn{1}{|c|}{ classe I } & $30-34,99$ \\
\multicolumn{1}{|c|}{ classe II } & $35-39,99$ \\
\multicolumn{1}{|c|}{ classe III } & $\geq 40$ \\
\hline
\end{tabular}

Nota: IMC= índice de massa corporal; Adaptado de OMS, 2003. 
Assim como uma série de doenças relacionadas ao sistema cardiovascular, a HAS pode ser prevenida por meio dos exercícios físicos e alimentação saudável, os quais auxiliam na manutenção do peso corporal normal e perfil lipídico plasmático favorável ${ }^{(53)}$. Esta informação se torna essencial uma vez que se conhece a relação existente entre peso corporal $^{(52,54)}$, triglicérides e colesterol sanguíneo com desenvolvimento de HAS ${ }^{(51)}$.

O exercício físico regular de intensidade baixa a moderada é recomendado como recurso essencial ao tratamento ${ }^{(52)}$, prevenção e controle da HAS ${ }^{(53)}$. O estilo de vida fisicamente ativo está descrito na literatura como forma de manutenção da saúde e níveis tensionais arteriais controlados ${ }^{(61,62)}$. A esse respeito, há razoável consenso de que maiores escores de atividade e aptidão física estejam associadas com redução de morbidades do aparelho cardiovascular e morte prematura ${ }^{(55)}$.

No entanto, os mecanismos pelos quais a atividade física diária e o exercício físico contribuem para manutenção dos valores de pressão arterial sistólica e diastólica mais baixos, ainda não estão totalmente esclarecidos. Estudos recentes apontam alguns caminhos e explicam em parte a fisiopatologia da doença e a ação da intervenção com exercício, contribuindo para o desenvolvimento de técnicas de tratamento mais eficazes ${ }^{(56,57)}$.

$\mathrm{O}$ esforço de pesquisadores ao redor do mundo tem caminhado no sentido de encontrar o nível de atividade física que possa contribuir para manter o indivíduo normotenso afastado do risco de desenvolver HAS, e por outro lado, no que diz respeito ao hipertenso, utilizar a técnica de melhor custo benefício, afastando-o da possibilidade de desenvolver patologias associadas.

\subsection{Tipos de Atividade Física.}


A definição clássica de atividade física proposta por Caspersen et al., ${ }^{(58)}$ é empregada por pesquisadores de todo o mundo desde sua publicação até os dias atuais e foi a utilizada neste trabalho como referência. A atividade física é conceituada como todo e qualquer movimento corporal produzido pelos músculos esqueléticos que resulte em gasto energético acima dos valores de repouso. No entanto, existem subdivisões, ou naturezas específicas enquadradas em diferentes categorias de atividade física.

Entre estas, a atividade física ocupacional (AFO) é entendida como as tarefas exercidas no período de trabalho. O gasto energético decorrente de atividades laborais pode ser determinante de saúde do organismo. Profissões como as tarefas de escritório, de motoristas, de costureiras, de telefonistas, entre outras, são exemplos de ocupações marcadamente sedentárias, com baixa demanda calórica. Em condição oposta, observa-se maior dispêndio de energia em ocupações consideradas pesadas, e que requerem caminhadas longas, como o serviço de entrega de correspondência nas residências. Tais atividades podem empregar melhor impacto sobre os componentes de saúde corporal relacionados à aptidão física.

Por atividade física de tempo livre (AFTL) entende-se como o oposto ao conceito anterior. São realizadas no período de lazer, principalmente aos finais de semana, quando se tem disponibilidade de tempo para aumentar o gasto energético apenas em períodos de "ociosidade". Esportes, recreação, passeios e caminhadas eventuais são exemplos desse tipo de atividade física. A AFTL independe da ocupação no trabalho e estas são somadas ao gasto total do indivíduo, promovendo melhores respostas em relação à saúde do organismo.

A atividade física de locomoção (AFL) é definida como as ações de deslocamento. O trajeto para o trabalho, principalmente nas grandes cidades dificilmente é realizada por meio de caminhadas ou com o uso de bicicletas, devido às maiores distâncias. Os órgãos 
públicos de saúde recomendam que as pessoas optem por deslocamentos a pé e pelo uso de escadas como alternativa para prevenir as doenças causadas pelo sedentarismo. No entanto, o que mais se observa é a adoção dos meios de transporte passivos (carros, ônibus, etc.).

O exercício físico (EF) é uma subcategoria de atividade física. Ambos têm o gasto energético acima dos níveis de repouso inclusos em suas características, porém, o que diferencia o EF é a necessidade de planejamento, repetição e estruturação como elementos fundamentais. Caracteriza-se como a situação que rompe com a homeostase do organismo, provocando ajustes e adaptações fisiológicas importantes ${ }^{(59)}$.

Outro aspecto que diferencia o exercício da atividade física é a sua utilização como forma de melhorar o nível de aptidão física. A aptidão relacionada à saúde possui componentes que são considerados atributos que a pessoa tem ou conquista por meio dos exercícios, e que a permite realizar tarefas diárias com vigor, sem fadiga, sobrando-lhe energia e vigor para os momentos de lazer ${ }^{(59)}$.

\subsection{Atividade Física e Saúde.}

A atividade física exerce papel fundamental sobre os componentes da saúde, uma vez que está inversamente relacionada com a presença de inúmeras enfermidades. O Ministério da Saúde ${ }^{(60)}$ aponta que $60 \%$ da população brasileira adulta que vive em áreas urbanas mantêm hábitos de atividade física insuficientes, condição que contribui para a preocupante projeção de que $73 \%$ dos casos de óbito em 2020 sejam atribuídos às doenças crônicas advindas do sedentarismo, sendo a hipertensão arterial uma das principais.

A esse propósito, vale destacar o estudo de coorte de Paffenbarguer ${ }^{(61)}$ quando observou que atividades de baixa intensidade, como tarefas domésticas, danças e passeios, 
acarretam redução significativa das manifestações das doenças coronárias. O nível de AF elevado é inversamente associado com mortalidade para doenças cardiovasculares, onde os incrementos em atividade diária foram associados com redução de peso e gordura intraabdominal ${ }^{(62)}$.

O estilo de vida sedentário é um comportamento que se associa também com perfil lipídico alterado, e ambos constituem fator de risco de diversas patologias, entre elas, a HAS. No estudo de Guedes e Gonçalves ${ }^{(27)}$, sujeitos ativos, avaliados por meio do International Physical Activity Questionnaire (IPAQ), apresentaram menores taxas de valores lipídicos plasmáticos, o que permite inferir um dos caminhos pelo qual a atividade física total do indivíduo exerça influência sobre a redução da pressão arterial.

As idéias expostas anteriormente corroboram com os dados amealhados no trabalho de Blair et al., ${ }^{(63)}$, que destaca os benefícios da atividade e a aptidão física para a saúde e redução da mortalidade e desenvolvimento de doenças do coração. A relação dose resposta tanto da atividade física como dos níveis de aptidão cardiorrespiratória mostrou-se inversamente associada com as taxas de mortalidade por todas as causas (ativos $v s$ sedentários; razão de 1:2).

Segundo Roberts e Bernard ${ }^{(64)}$, as evidências científicas produzidas nos últimos vinte anos têm documentado a reversão da progressão de doenças crônicas pela ação da $\mathrm{AF}$ e dieta, combinadas ou não. Entre os benefícios da AF na redução de DCV são descritos efeitos concomitantes, também sobre o perfil lipidico ${ }^{(65)}$, função endotelial ${ }^{(66)}$ e resistência à insulina ${ }^{(67)}$. O Women'S Health Initiative Observational Study (1994-1998) investigou a relação entre AF (caminhada e exercícios vigorosos) e DCV. Os resultados obtidos 
mostraram que médias superiores a 2,5 horas semanais dedicadas à caminhadas, foram responsáveis por redução de 30 a $40 \%$ das $\operatorname{DCV}^{(68)}$.

Diversas diretrizes internacionais ${ }^{(69-72)}$ foram publicadas propondo o aumento da atividade física diária e dos exercícios regulares (ao menos 30 minutos por dia) associados à alimentação balanceada, como formas prevenção de doenças e manutenção das condições de saúde. Nestas comunicações a AF é proposta como a alternativa mais adequada para a prevenção e controle de morbidades crônico-degenerativas resultantes do comportamento sedentário, por promover manutenção do peso corporal e ajustes importantes no sistema cardiovascular. Estas iniciativas são importantes quando se considera que os custos com a terapêutica medicamentosa empregada junto aos pacientes em tratamento são demasiadamente altos ${ }^{(73)}$, mas de modo geral, particularmente em relação à hipertensão arterial, não exercem efeito curativo, apenas controle dos níveis tensionais.

Contudo, ainda há necessidade de determinar o nível ideal de atividade física capaz de promover benefícios ao organismo, afastando o risco de desenvolvimento de patologias associadas ao sedentarismo. Nesse contexto, há estudos buscando entender as relações do nível de atividade física sobre a manutenção da saúde, sendo as diversas naturezas em que ela se ramifica, o tema das investigações. Denominadas atividade física de tempo livre (AFTL), ocupacional (AFO) e de locomoção (AFL) elas têm sido estudadas para identificar as ações reais que exercem e por meio de quais mecanismos elas contribuem para reduzir a incidência de doenças.

\subsection{Nível de Atividade Física e Hipertensão Arterial.}

Estudos epidemiológicos têm encontrado menor prevalência e incidência de HAS e demais morbidades ligadas ao aparelho cardiovascular entre indivíduos com maiores níveis de atividade física diária ${ }^{(74,75)}$. 
Blair et al., ${ }^{(63)}$ demonstraram por meio de estudo de coorte de quatro anos, que o risco relativo para o desenvolvimento da doença é maior em inativos, comparativamente aos ativos. A doença também está associada a outros fatores negativos do estilo de vida, como consumo de álcool e tabagismo, já descritos neste trabalho como fatores de risco. Swartz et al., ${ }^{(77)}$, afirmam que indivíduos classificados como fisicamente ativos pela quantidade de passos diários e com IMC normal, comparados com obesos inativos, têm valores estatisticamente menores de PAS $(138,8$ vs $123,9 \mathrm{mmHg}: \mathrm{p}<0,05)$ e PAD $(81,5$ vs 75,7mmHg: $\mathrm{p}<0,05)$.

Estudo longitudinal realizado no Japão analisou a relação entre o tempo gasto com locomoção para o trabalho e incidência de HAS ${ }^{(78)}$. Os resultados da pesquisa realizada com 6104 homens com idade superior a 35 anos encontraram relação inversa entre AFL e hipertensão e, efeito protetor da caminhada com duração acima de 21 minutos. Os resultados mostraram redução de $12 \%$ no risco para HAS com o acréscimo de 10 minutos no tempo da caminhada diária ao trabalho.

Wagner et al., ${ }^{(79)}$ observaram que o gasto energético de atividade física de tempo livre (AFTL) foi associado com menor risco de eventos cardiovasculares $(\mathrm{p}<0,05)$, enquanto que AFL não mostrou ter a mesma relação. Outros pesquisadores sugerem que as relações do nível de AFTL e PA são menos consistentes do que associações com indicadores biológicos ${ }^{(80)}$.

Dados recentes apontam divergências, possivelmente pela variedade nos métodos de mensuração da AF e conseqüente interpretação do custo energético das atividades. As pesquisas que buscaram identificar se atividades de lazer ou ocupacionais têm impacto sobre variáveis hemodinâmicas e metabólicas, mostram resultados conflitantes ${ }^{(11,15,81 ;)}$, ou pouco expressivos ${ }^{(16)}$. No estudo de Sobngwi et al., ${ }^{(82)}$, por exemplo, a AFO não se 
associou com a HAS. Na investigação de Niskanen et al., ${ }^{(51)}$ não foram encontradas associações da AFTL com a incidência de HAS em um acompanhamento de 11 anos.

Entre os diferentes tipos de atividades físicas e sua relação com pressão arterial, é preciso considerar também as diferenças entre sexos. Neste caso, também encontram-se divergências nos resultados dos trabalhos. O estudo de Oppert et al., ${ }^{(83)}$ mostrou associação positiva para AFO com a PAS $(\mathrm{p}=0,0007)$ e PAD $(\mathrm{p}=0,02)$ apenas para os homens. Há indicação, ainda por meios desses dados de que a AFTL seja um comportamento saudável para reduzir o risco de DCV. Contrariamente, $\mathrm{Hu}$ et al., ${ }^{(1)}$ encontraram associação entre níveis moderados ou elevados de AFO e AFTL com o menor risco de DCV para as mulheres. Neste caso, sugerem os autores que as atividades de tempo livre e as ocupacionais parecem apenas atenuar, mas não eliminar a associação inversa entre fatores de risco e incidência de DCV. Por fim, Apullan et al., ${ }^{(84)}$ encontraram resultados similares para homens e mulheres em relação à atividade física de tempo livre associada ao risco de morte.

A importância de pesquisas dessa natureza considera a relação custo-benefício do exercício físico sistematizado comparado a outros tipos de atividade física. Nos esportes, por exemplo, tem-se maior incidência de lesões osteoarticulares e musculares, o que, com o avanço da idade, torna-se fator que se contrapõe à adesão ao estilo de vida fisicamente ativo. A identificação dessa resposta torna-se de grande relevância, principalmente em âmbito populacional. Barengo et al., ${ }^{(81)}$ afirmam, baseados em estudos de revisão, a necessidade de mais investigações acerca das atividades ocupacionais e de vida diária que possam resultar em menores valores pressóricos e proteção ao organismo.

A determinação da AF em levantamentos populacionais geralmente é efetuada por meio de questionários ${ }^{(2)}$. Para este fim existem instrumentos validados na população 
brasileira com boa reprodutibilidade e confiabilidade, além do que se constituem em formas bastante utilizadas por representarem baixo custo e facilidade de aplicação.

Em levantamento epidemiológico nacional tendo a atividade física como variável de investigação, Hallal et al., ${ }^{(85)}$ identificaram 42 estudos originais que utilizaram mais de 500 indivíduos na amostra (desde1990 quando o primeiro deles foi publicado). Nestas pesquisas 93\% empregaram questionários, das quais, $69 \%$ foram desenvolvidas pelos próprios pesquisadores, e apenas 7\% utilizaram o IPAQ como instrumento de estimativa do nível de atividade física.

Apesar de se reconhecer as limitações metodológicas existentes em adotar este procedimento, os questionários destacam-se por obter informações de forma rápida, não invasiva e de baixo custo e que, de modo geral quando aplicados com rigor produzem resultados confiáveis ${ }^{(86-89)}$.

\section{MATERIAL E MÉTODOS}

\subsection{Natureza da pesquisa, casuística e local de estudo}

A pesquisa se caracteriza como estudo epidemiológico de corte (tipo transversal) com componente retrospectivo a partir da coleta de dados.

De acordo com estimativa da divisão regional de saúde 10 do Estado de São Paulo, a região de Bauru possui cerca de 17 mil pessoas acometidas por HAS. O presente trabalho foi realizado junto aos Núcleos de Saúde "Otávio Rasi” e "Vila Cardia”, ambos localizados na região sudoeste da cidade de Bauru. A escolha destes bairros se deve ao fato de serem núcleos acompanhados por projeto da Faculdade de Ciências, Unesp campus de Bauru, e encontra-se no sexto ano de execução. As intervenções realizadas por tais projetos foram aprovadas pelo comitê de ética da secretaria municipal de saúde. 
A população local total acometida por HAS, somando-se os dois locais, constitui universo de aproximadamente 835 pessoas, contando tanto entre os matriculas que são faltosos às sessões de monitoramento da PA e, aqueles que comparecem regularmente para fazer o acompanhamento médico (uma vez ao mês). De toda a população que compareceu aos postos nos dias de coleta, que tinham hipertensão diagnosticada há pelo menos um ano confirmada por meio do prontuário e ainda, estavam sob tratamento farmacológico, 192 pacientes aceitaram participar da pesquisa (todos maiores que 18 anos de idade). Para caracterização do perfil dos pacientes foi realizada entrevista dirigida (anamnese) contendo perguntas sobre a condição sócio-econômica, etnia, sexo, estado civil e hábitos do estilo de vida (tabagismo e consumo de álcool) (ANEXOS 1 e 2). Todos pacientes foram entrevistados individualmente, por um único avaliador a fim de minimizar diferenças entre pesquisadores nos resultados obtidos.

\subsection{Variáveis do estudo}

\subsubsection{Nível de atividade física}

Questionários para identificação do NAF são utilizados para determinar o ponto a partir do qual o exercício promove benefícios ao organismo, seja como coadjuvante no tratamento de doenças crônicas, ou, como fator de prevenção para o seu surgimento ${ }^{(11,128,}$ 129). Pela facilidade de aplicação e baixo custo, eles são considerados bons instrumentos, principalmente em estudos com grandes populações ${ }^{(2,90)}$.

O NAF dos participantes da presente pesquisa foi estimado por meio do questionário de Baecke et al., ${ }^{(90)}$, validado nacionalmente primeiramente com adultos ${ }^{(91)}$ e, mais recentemente com adolescentes ${ }^{(92)}$. Sua utilização em pesquisas internacionais ${ }^{(93,94)}$ e nacionais ${ }^{(95-97)}$ apresentou reprodutibilidade e validade satisfatórias ${ }^{(98-100)}$. O instrumento 
é de fácil entendimento para pessoas que se encontram na faixa etária superior a 50 anos, mas ainda assim foi aplicado em formato de entrevista.

No referido questionário as atividades são categorizadas em ocupacionais (AFO), esportivas e de tempo livre (AFTL), e de locomoção (AFL). A soma dos escores finais de todas as naturezas de AF (AFO + AFTL + AFL) foi adotada neste trabalho como “Atividade Física Total” (AF Total) (ANEXO 3).

O questionário apresenta valor de escala adimensional para cada categoria de atividade física, com as pontuações em cada questão variando de 1 a 5 , os quais são utilizadas para o cálculo dos escores finais de atividade física em cada categoria por meio de equações propostas por Baecke et al., ${ }^{(90)}$ no artigo original do questionário.

Por não existir uma classificação para os valores finais de escore do questionário os quais enquadrem os sujeitos avaliados em grupos, segundo nível de atividade física (sedentários, moderadamente ativos e ativos), foi adotado, na presente pesquisa, que em cada categoria de AF, fosse calculado o valor médio do escore do grupo e, posteriormente, o conjunto de pacientes que apresentaram escores acima da média foi tratado como ativos (AT), enquanto aqueles com escores abaixo da média, sedentários (SED) (Quadro 3).

Quadro 3. Número de pacientes em cada grupo de acordo com o nível de atividade física em cada categoria.

\begin{tabular}{|lcc|}
\hline & \multicolumn{2}{c|}{ Nível de Atividade Física } \\
\cline { 2 - 3 } Categoria de AF & Ativo (n) & Sedentário (n) \\
\hline Ocupacional (AFO) & 92 & 100 \\
Tempo Livre (AFTL) & 68 & 124 \\
Locomoção (AFL) & 74 & 118 \\
Total (AFT) & 84 & 108 \\
\hline
\end{tabular}




\subsubsection{Distúrbios metabólicos, cardiovasculares e osteomusculares.}

Estudos epidemiológicos têm apontado boa concordância para questionários de doenças auto-referidas e entrevistas com prontuários médicos ${ }^{(101)}$, principalmente para o diabetes ${ }^{(102,}{ }^{103)}$, hipertensão e moléstias do coração ${ }^{(104)}$. Estes instrumentos, principalmente quando aplicados sob a forma de entrevista, são ferramentas adequadas para a obtenção de dados relativos ao histórico e presença de doenças crônicas e osteomusculares.

Devido à ausência de protocolo amplamente utilizado e validado para avaliação de doenças referidas, um novo inquérito de doenças, adaptado a partir de outros encontrados na literatura ${ }^{(105)}$, foi adotado para a aplicação nesta população em formato de entrevista (ANEXO 2). Os pacientes foram interrogados sobre a presença de doenças crônicas como diabetes, cardiovasculares, distúrbios metabólicos e osteomusculares, entre outras condições mórbidas que pudessem ser julgadas relevantes.

As moléstias referidas pelos doentes na entrevista foram reunidas em três grupos distintos, de acordo com a Classificação Estatística Internacional de Doenças e Problemas Relacionado à Saúde - CID ${ }^{(106)}$ da Organização Mundial de Saúde e Organização Panamericana de Saúde: a) Doenças endócrinas, nutricionais e metabólicas (Intervalo CID= E00 - E90), sendo elas: diabetes, colesterol alto, hiper e hipotiroidismo; b) Doenças do aparelho circulatório (Intervalo CID= I00 - I99): arritmia, infarto e angina; c) Doenças do sistema osteomuscular e do tecido conjuntivo (Intervalo CID= M00 - M99): osteoporose, artrite, artrose, lombalgias e hérnia de disco.

Patologias raramente relatadas pelos pacientes, como o câncer (relatada uma única vez), não foram computadas e classificadas para as análises. 


\subsubsection{Avaliação do paciente}

\subsubsection{Ao exame}

As medidas de massa corporal total e a estatura foram utilizadas para o cálculo do Índice de Massa Corporal (IMC, $\mathrm{kg} / \mathrm{m}^{2}$ ), sendo os procedimentos de medida adotados, os descritos por Lohman et al., ${ }^{(107)}$, sempre efetuadas pelo mesmo avaliador.

A medida de Circunferência de Cintura (CC) foi utilizada como índice de caracterização antropométrica em relação ao padrão de distribuição corporal, empregada como fator de risco para HAS, e possíveis associações distúrbios metabólicos e demais doenças referidas na entrevista. Para este procedimento foi utilizada fita antropométrica com precisão de $0,1 \mathrm{~cm}$, no ponto da menor circunferência entre a crista ilíaca e a última costela flutuante ${ }^{(108)}$. Os valores de corte para caracterização de risco segundo a circunferência de cintura foram: $>102 \mathrm{~cm}$ para homens e $>88 \mathrm{~cm}$ para mulheres.

A pressão arterial de repouso foi aferida utilizando-se esfigmomanômetro de coluna de mercúrio, seguindo as recomendações estabelecidas pela V DBHA ${ }^{(1)}$.

Ao final das avaliações, os fatores de risco levantados foram classificados conforme categorização que pode ser visualizada no Quadro 4.

Quadro 4. Classificação dos dados sócio-demográficos segundo fatores de risco e de proteção.

\begin{tabular}{|lccc|}
\hline Fator & Escolaridade & Etnia & Estado Civil \\
\hline Risco & $<$ anos & negra, parda, & solteiro, \\
\multirow{2}{*}{ Proteção } & $>$ anos & amarela & divorciado, viúvo \\
\hline
\end{tabular}




\subsection{Aspectos éticos}

Os pacientes que concordaram em participar da pesquisa foram previamente informados sobre os procedimentos de avaliação e condições para participação e, posteriormente, assinaram termo de consentimento livre e esclarecido aprovado pelo comitê de ética em pesquisa da Faculdade de Ciências e Tecnologia, Unesp - Campus de Presidente Prudente (Processo n 322-08), autorizando a utilização das informações para fins científicos, assegurando-lhes o sigilo e a preservação de sua identidade.

\subsection{Análise estatística.}

Para as variáveis numéricas, anteriormente às análises, o teste de KomolgorovSmirnov foi empregado e indicou que as variáveis apresentaram distribuição normal, assim, procedimentos paramétricos foram empregados.

Para comparar proporções e verificar existência de associação entre variáveis categóricas foi utilizado o Teste Qui-quadrado. As comparações entre grupos foram feitas por meio do teste $T$ de Student, para dados não pareados. O teste de correlação de Pearson foi empregado para associações entre valores de pressão arterial e os escores de cada categoria de atividade física.

O nível de significância foi previamente estabelecido em 5\%, e todas as análises foram feitas utilizando o software estatístico SPSS versão 13.0.

\section{RESULTADOS}

\subsection{Perfil da casuística.}

A Tabela 1 apresenta as médias das variáveis antropométricas, pressão arterial sistólica e diastólica e dos escores de cada categoria de atividade física da casuística como 
um todo ( $\mathrm{n}=192)$, e separada por sexo. Observa-se prevalência de sobrepeso (IMC) e risco coronariano indicado pela $\mathrm{CC}$, tanto para homens $(>102 \mathrm{~cm})$ como para mulheres $(>88 \mathrm{~cm})$. Embora se trate de população hipertensa, as pressões sistólica e diastólica da casuística apresentam-se abaixo dos valores considerados diagnóstico para hipertensão arterial segundo a V DBHA ${ }^{(1)}$.

Quanto aos escores de atividade física, a categoria que apresentou média mais elevada foi a ocupacional $(2,37 \pm 0,77)$, seguida da atividade física de tempo livre $(2,01 \pm$ $1,43)$ e de locomoção $(1,55 \pm 0,54)$. Estratificando por sexo, para as mulheres é apresentada a mesma tendência que do grupo em geral, porém para os homens a AFTL apresentou média de escore maior que a $\mathrm{AFO}(2,44$ vs 2,27). Em relação à atividade física total, os homens apresentaram média de escore mais elevado em comparação às mulheres $(6,24 \mathrm{vs}$ $5,78)$.

Tabela 1. Valores descritivos de média e desvio padrão para variáveis antropométricas, pressão arterial e escores das categorias de atividade física.

\begin{tabular}{lccccc}
\hline & $\begin{array}{c}\text { Casuística } \\
\mathrm{n}=192\end{array}$ & \multicolumn{2}{c}{$\begin{array}{c}\text { Mulheres } \\
\mathrm{n}=132\end{array}$} & \multicolumn{2}{c}{$\begin{array}{c}\text { Homens } \\
\mathrm{n}=60\end{array}$} \\
\cline { 2 - 6 } Variável & Média e DP & Média & DP & Média & DP \\
\hline Idade (anos) & $63 \pm 11$ & 62 & 11 & 63 & 20 \\
Peso $(\mathrm{kg})$ & $77,15 \pm 19,56$ & 73,46 & 17,41 & 85,13 & 21,62 \\
IMC $\left(\mathrm{kg} / \mathrm{m}^{2}\right)$ & $29,75 \pm 6,43$ & 29,78 & 6,53 & 29,70 & 6,26 \\
CC $(\mathrm{cm})$ & $99,14 \pm 16,28$ & 96,79 & 13,55 & 104,22 & 20,24 \\
PAS $(\mathrm{mmHg})$ & $133,61 \pm 15,18$ & 132,85 & 16,16 & 135,28 & 12,71 \\
PAD (mmHg) & $81,04 \pm 10,96$ & 81,30 & 10,76 & 80,48 & 11,45 \\
\hline Atividade Física & & & & & \\
Ocupacional & $2,37 \pm 0,77$ & 2,41 & 0,73 & 2,27 & 0,85 \\
Tempo Livre & $2,01 \pm 1,43$ & 1,82 & 1,22 & 2,44 & 1,74 \\
Locomoção & $1,55 \pm 0,54$ & 1,56 & 0,55 & 1,53 & 0,52 \\
Total & $5,92 \pm 1,76$ & 5,78 & 0,67 & 6,24 & 1,92 \\
\hline
\end{tabular}

Nota: IMC $=$ índice de massa corporal; $\mathrm{CC}=$ circunferência de cintura; $\mathrm{PAS}=$ pressão arterial sistólica; $\mathrm{PAD}=$ pressão arterial diastólica; $\mathrm{DP}=$ desvio padrão. 
A Tabela 2 apresenta o perfil da casuística como um todo e dividida por sexo, distribuída em relação aos dados demográficos. Observou-se que a maioria dos participantes é do sexo feminino $(\mathrm{n}=132)$, possui escolaridade referente ao primário ao ginásio incompleto $(50,0 \%)$, pertence à etnia branca $(61,5 \%)$, e são casados $(55,7 \%)$.

Tabela 2. Distribuição da freqüência dos pacientes estudados, segundo caracterização e sexo.

\begin{tabular}{lcccc}
\hline Variável & $\mathrm{N}$ & $(\%)$ & Mulheres $(\%)$ & Homens $(\%)$ \\
\hline Escolaridade & & & & \\
\hline Primário incompleto & 49 & 25,5 & 22,7 & 31,7 \\
Ginásio incompleto & 96 & 50,0 & 58,3 & 31,7 \\
Colegial incompleto & 15 & 7,8 & 8,3 & 6,7 \\
Superior incompleto & 27 & 14,1 & 8,3 & 26,7 \\
Superior completo & 5 & 2,6 & 2,3 & 3,3 \\
\hline Etnia & & & & 70,0 \\
Branca & 118 & 61,5 & 57,6 & 28,3 \\
Negra & 59 & 30,7 & 31,8 & 1,7 \\
Amarela & 6 & 3,1 & 3,8 & -- \\
Parda & 9 & 4,7 & 6,8 & 15,0 \\
\hline Estado Civil & & & & 73,3 \\
Solteiro & 34 & 17,7 & 18,9 & 3,3 \\
Casado & 107 & 55,7 & 47,7 & 8,3 \\
$\quad$ Divorciado & 18 & 9,4 & 12,1 & \\
Viúvo & 32 & 16,7 & 20,5 & \\
\hline
\end{tabular}

\subsection{Nível de atividade física.}

O perfil da casuística segundo o nível de atividade física, dividida por categoria das atividades (ocupacional, tempo livre, de locomoção e AF Total) é apresentado na Tabela 3. Comparando as médias apresentadas na tabela 3, e excluindo-se a AF Total que representa a soma de todos os outros escores, a atividade física de tempo livre apresenta o maior escore entre os ativos $(3,48 \pm 1,50)$, seguida pela ocupacional $(3,02 \pm 0,54)$, e de 
locomoção $(2,10 \pm 0,42)$. Entre os sedentários observa-se a atividade física ocupacional como a de escore mais elevado $(1,76 \pm 0,33)$ em comparação às demais categorias.

Tabela 3. Valores de média e desvio padrão dos escores de atividade física divididos por categoria e níveis de atividade física.

\begin{tabular}{ccc}
\hline Categoria de & \multicolumn{2}{c}{ Nível de Atividade Física } \\
\cline { 2 - 3 } Atividade Física & Ativo & Sedentário \\
\hline Ocupacional & $3,02 \pm 0,54$ & $1,76 \pm 0,33^{*}$ \\
Tempo Livre & $3,48 \pm 1,50$ & $1,20 \pm 0,32^{*}$ \\
Locomoção & $2,10 \pm 0,42$ & $1,20 \pm 0,24^{*}$ \\
Total & $7,49 \pm 1,42$ & $4,70 \pm 0,74^{*}$ \\
\hline
\end{tabular}

(*) Teste T de Student.

A Tabela 4 apresenta os valores de média e desvio padrão da casuística para a idade, variáveis antropométricas e pressão arterial dentro de cada categoria de atividade física separada segundo os grupos ativo e sedentário. Notam-se diferenças estatísticas significantes $(\mathrm{p}<0,05)$ em algumas variáveis, comparando-se os grupos. As categorias AFL e AFO não exerceram influência sobre as variáveis em questão, apresentando médias semelhantes entre os grupos, com exceção para a variável idade dentro da categoria AFO. Para a AFTL observam-se diferenças significantes para as variáveis PAS $(130,19 \mathrm{mmHg}$ vs $135,48 \mathrm{mmHg} ; \mathrm{p}=0,02)$ e PAD $(78,21 \mathrm{mmHg}$ vs $82,60 \mathrm{mmHg} ; \mathrm{p}=0,01)$ onde os indivíduos ativos apresentam valores médios inferiores aos sedentários. Finalizando, a AF Total exerceu influência sobre PAS e PAD, onde os valores médios mais elevados são observados para o grupo $\mathrm{SED}(\mathrm{PAS}=136,85 \mathrm{mmHg} ; \mathrm{PAD}=82,56 \mathrm{mmHg}$, quando comparados aos AT (PAS=129,44mmHg; PAD=79,08mmHg). 
Tabela 4. Média e desvio padrão dos dados antropométricos, pressão arterial e idade, dividido por categoria da atividade física.

\begin{tabular}{lcccccc}
\hline Atividade & $\begin{array}{c}\text { Idade } \\
(\text { anos })\end{array}$ & $\begin{array}{c}\text { Peso } \\
(\mathrm{Kg})\end{array}$ & $\begin{array}{c}\mathrm{IMC} \\
\left(\mathrm{Kg} / \mathrm{m}^{2}\right)\end{array}$ & $\begin{array}{c}\mathrm{CC} \\
(\mathrm{cm})\end{array}$ & $\begin{array}{c}\text { PAS } \\
(\mathrm{mmHg})\end{array}$ & $\begin{array}{c}\text { PAD } \\
(\mathrm{mmHg})\end{array}$ \\
\hline $\begin{array}{l}\text { Ocupacional } \\
\quad \text { AT }\end{array}$ & $59 \pm 10$ & $76,34 \pm 19,15$ & $30,00 \pm 6,92$ & $97,09 \pm 14,58$ & $132 \pm 15,20$ & $81,91 \pm 11,04$ \\
$\quad \mathrm{SED}$ & $66 \pm 10$ & $77,87 \pm 19.99$ & $29,53 \pm 5,98$ & $100,98 \pm 17,54$ & $134 \pm 15,11$ & $80,24 \pm 10,88$ \\
\hline $\mathrm{t}(\mathrm{P}=)$ & $\mathbf{4 , 8 8 ( \mathbf { 0 , 0 0 1 } )}$ & $0,54(0,59)$ & $-0,50(0,61)$ & $1,65(0,10)$ & $1,24(0,21)$ & $-1,05(0,29)$ \\
\hline
\end{tabular}

Tempo Livre

AT $\quad 64 \pm 10 \quad 79,55 \pm 19,77 \quad 30,03 \pm 6,27 \quad 100,27 \pm 17,85 \quad 130,19 \pm 13,95 \quad 78,21 \pm 8,94$

SED $\quad 62 \pm 11 \quad 75,84 \pm 19,40 \quad 29,60 \pm 6,52 \quad 95,35 \pm 15,30 \quad 135,48 \pm 15,54 \quad 82,60 \pm 11,67$

\begin{tabular}{lcccccc}
\hline $\mathrm{t}(\mathrm{P}=)$ & $-1,51(0,13)$ & $1,25(0,21)$ & $-0,45(0,64)$ & $-1,40(0,16)$ & $\mathbf{2 , 3 3}(\mathbf{0 , 0 2})$ & $\mathbf{2 , 6 9}(\mathbf{0 , 0 1})$ \\
\hline Locomoção & & & & & & \\
$\quad \mathrm{AT}$ & $63 \pm 10$ & $74,81 \pm 14,17$ & $29,13 \pm 5,56$ & $96,82 \pm 13,56$ & $131,05 \pm 13,61$ & $79,92 \pm 9,75$ \\
$\mathrm{SED}$ & $63 \pm 11$ & $78,64 \pm 22,26$ & $30,16 \pm 6,92$ & $100,62 \pm 17,70$ & $135,21 \pm 1593$ & $81,75 \pm 11,64$ \\
\hline $\mathrm{t}(\mathrm{P}=)$ & $0,05(0,96)$ & $1,31(0,19)$ & $1,08(0,28)$ & $1,58(0,11)$ & $1,86(0,06)$ & $1,13(0,26)$ \\
\hline
\end{tabular}

AF Total

AT $\quad 61 \pm 10 \quad 77,69 \pm 16,72 \quad 29,74 \pm 6,06 \quad 98,45 \pm 16,78 \quad 129,44 \pm 13,07 \quad 79,08 \pm 8,71$

SED $\quad 64 \pm 11 \quad 76,74 \pm 21,53 \quad 29,77 \pm 6,71 \quad 100,04 \pm 15,65 \quad 136,85 \pm 15,95 \quad 82,56 \pm 12,25$

\begin{tabular}{lllllll}
$\mathrm{t}(\mathrm{P}=)$ & $1,54(0,26)$ & $0,33(0,74)$ & $0,03(0,97)$ & $0,67(0,50)$ & $\mathbf{3 , 4 5}(\mathbf{0 , 0 0 1})$ & $\mathbf{1 0 , 3 3}(\mathbf{0 , 0 0 2})$ \\
\hline
\end{tabular}

Nota: Teste $T$ de Student; $\mathrm{AT}=$ Ativo; $\mathrm{SED}=$ Sedentário; $\mathrm{IMC}=$ índice de massa corporal; $\mathrm{CC}=$ circunferência de cintura; $\mathrm{PAS}=$ pressão arterial sistólica; $\mathrm{PAD}=$ pressão arterial diastólica.

A Tabela 5 apresenta os valores do índice beta ( $\beta$ ) entre os escores de atividade física para cada categoria estudada e as variáveis idade e valores de pressão arterial, ajustados pelos fatores de risco: idade, escolaridade, etnia, sexo, estado civil e risco cardiovascular (CC). Observa-se correlação negativa significante entre a variável idade com os escores de atividade física ocupacional e total. A pressão arterial sistólica correlacionou-se negativamente com as atividades físicas: de locomoção $(r=-0,17)$ e total $(r=-0,19)$. 
Tabela 5. Regressão linear ( $\beta$ ) entre variáveis antropométricas, idade e pressão arterial, com escore de atividade física.

\begin{tabular}{lcccccc}
\hline Atividade & Idade & Peso & IMC & CC & PAS & PAD \\
\hline Ocupacional & $\mathbf{- 0 , 0 2 * *}$ & 0,00 & 0,00 & $-0,00$ & $-0,00$ & $-0,00$ \\
Tempo Livre & $-0,00$ & $-0,00$ & $-0,01$ & $-0,00$ & $-0,01$ & $-0,00$ \\
Locomoção & $-0,00$ & 0,00 & $-0,00$ & $-0,00$ & $\mathbf{- 0 , 0 0 *}$ & $-0,00$ \\
AF Total & $\mathbf{- 0 , 0 2 * *}$ & $-0,00$ & $-0,01$ & $-0,01$ & $\mathbf{- 0 , 0 2 * *}$ & $-0,00$
\end{tabular}

Nota: Correlação de Pearson; $*=\mathrm{p}<0,05 ;{ }^{* *}=\mathrm{p}<0,01 ; \mathrm{IMC}=$ índice de massa corporal;

$\mathrm{CC}=$ circunferência de cintura; $\mathrm{PAS}=$ pressão arterial sistólica; $\mathrm{PAD}=$ pressão arterial diastólica.

\subsection{Presença de doenças.}

O perfil da casuística segundo presença dos fatores de risco e dividida entre os três grupos de doenças categorizados segundo classificação da CID é apresentado na Tabela 6. O Odds Ratio foi calculado para cada fator de risco, em relação às doenças referidas. Notase a influência do fator escolaridade sobre a categoria de doenças CID- M00-M99 (doenças osteomusculares e do tecido conjuntivo), onde os pacientes com escolaridade $>8$ exibem fator de proteção contra esses tipos de moléstias e o sexo feminino apresentou fator de risco $(\mathrm{OR}=2,68 ; \mathrm{IC}=1,42-5,06)$. Do mesmo modo, a ausência do hábito alcoólico para esta categoria de doenças representou fator de proteção ( $\mathrm{OR}=0,33 ; \mathrm{IC}=0,11-0,96)$. Entre as doenças do aparelho circulatório os homens apresentaram fator de proteção em relação às mulheres $(\mathrm{OR}=0,40 ; \mathrm{IC}=0,21-0,77)$. 
Tabela 6. Distribuição de acordo com fatores de risco em relação presença de doenças.

\begin{tabular}{|c|c|c|c|c|c|c|}
\hline \multirow[b]{3}{*}{ Fator de Risco } & \multicolumn{6}{|c|}{ Classificação Internacional de Doenças } \\
\hline & \multicolumn{2}{|c|}{ E00 - E90 } & \multicolumn{2}{|c|}{ I00 - I99 } & \multicolumn{2}{|c|}{ M00 - M99 } \\
\hline & $\mathrm{n}$ & $\%$ & $\mathrm{n}$ & $\%$ & $\mathrm{n}$ & $\%$ \\
\hline \multicolumn{7}{|l|}{ Escolaridade } \\
\hline$>8$ anos & 32 & 68,1 & 19 & 40,4 & 13 & 27,7 \\
\hline$<8$ anos & 82 & 56,6 & 38 & 26,2 & 86 & 59,3 \\
\hline TO $(\mathrm{IC}-95 \%)$ & \multicolumn{2}{|c|}{$1,64(0,82-3,29)$} & \multicolumn{2}{|c|}{$1,91(0,96-3,81)$} & \multicolumn{2}{|c|}{$0,26(0,13-0,54)$} \\
\hline \multicolumn{7}{|l|}{ Etnia } \\
\hline Outras & 38 & 51,4 & 24 & 32,4 & 37 & 50,0 \\
\hline Branca & 76 & 64,4 & 33 & 28,1 & 62 & 52,5 \\
\hline TO $(\mathrm{IC}-95 \%)$ & \multicolumn{2}{|c|}{$0,58(0,32-10,5)$} & \multicolumn{2}{|c|}{$1,24(0,66-2,32)$} & \multicolumn{2}{|c|}{$0,90(0,50-1,62)$} \\
\hline \multicolumn{7}{|l|}{ Estado Civil } \\
\hline Outros & 46 & 54,1 & 21 & 24,7 & 46 & 54,1 \\
\hline Casado & 68 & 63,0 & 36 & 33,6 & 53 & 49,5 \\
\hline TO (IC - 95\%) & \multicolumn{2}{|c|}{$0,68(0,38-1,21)$} & \multicolumn{2}{|c|}{$0,65(0,35-1,26)$} & \multicolumn{2}{|c|}{$1,20(0,68-2,13)$} \\
\hline \multicolumn{7}{|l|}{ Fumo } \\
\hline Presente & 08 & 42,1 & 08 & 42,1 & 08 & 42,1 \\
\hline Ausente & 106 & 61,3 & 49 & 28,3 & 91 & 52,6 \\
\hline$T O(\mathrm{IC}-95 \%)$ & \multicolumn{2}{|c|}{$0,46(0,18-1,20)$} & \multicolumn{2}{|c|}{$1,84(0,70-4,85)$} & \multicolumn{2}{|c|}{$0,66(0,25-1,71)$} \\
\hline \multicolumn{7}{|l|}{ Álcool } \\
\hline Presente & 09 & 50,0 & 08 & 44,4 & 05 & 27,8 \\
\hline Ausente & 105 & 60,3 & 49 & 28,2 & 94 & 54,0 \\
\hline TO (IC - 95\%) & \multicolumn{2}{|c|}{$0,66(0,25-1,74)$} & \multicolumn{2}{|c|}{$2,04(0,76-5,47)$} & \multicolumn{2}{|c|}{$0,33(0,11-0,96)$} \\
\hline \multicolumn{7}{|l|}{$\mathrm{CC}$} \\
\hline Presente & 90 & 62,9 & 46 & 32,2 & 72 & 50,3 \\
\hline Ausente & 24 & 49,0 & 11 & 22,4 & 27 & 55,1 \\
\hline TO (IC - 95\%) & \multicolumn{2}{|c|}{$1,77(0,92-3,40)$} & \multicolumn{2}{|c|}{$1,64(0,77-3,49)$} & \multicolumn{2}{|c|}{$0,83(0,43-1,59)$} \\
\hline
\end{tabular}

Nota: TO- Taxa de Odds; Classificação Internacional de Doenças (CID)= CID E00-E90: Doenças endócrinas, nutricionais e metabólicas; CID I00-I99: Doenças do aparelho circulatório; CID M00M99: Doenças do sistema osteomuscular e do tecido conjuntivo; $\mathrm{CC}=$ circunferência de cintura.

Em seguida, dentro de cada categoria de atividade física separado entre ativos e sedentários, os pacientes foram distribuídos entre os grupos de doenças CID (Tabela 7).

Para a AFO e AFL não são observadas relações entre nível de atividade física e as doenças para todas as categorias da CID. Para a AFTL, percebe-se predominância de doenças endócrino-metabólicas (CID E00-E90) entre os indivíduos com maior nível de 
atividade física apresentaram 2,36 vezes maior chance de apresentar este tipo de moléstia $(\mathrm{TO}=2,36 ; \mathrm{IC}=1,18-4,70)$. Do mesmo modo, a atividade física total exibe fator de risco dentro da mesma categoria de doenças (CID E00-E90), apresentando os ativos, 2,5 vezes mais chance de serem acometidos que os sedentários.

Tabela 7. Distribuição da casuística de acordo com nível de atividade física entre os grupos de doenças (CID).

\begin{tabular}{|c|c|c|c|c|c|c|}
\hline \multirow[b]{3}{*}{ Atividade Física } & \multicolumn{6}{|c|}{ Classificação Internacional de doenças - CID } \\
\hline & \multicolumn{2}{|c|}{ E00 - E90 } & \multicolumn{2}{|c|}{ I00 - I99 } & \multicolumn{2}{|c|}{ M00 - M99 } \\
\hline & $\mathrm{n}$ & $\%$ & $\mathrm{n}$ & $\%$ & $\mathrm{n}$ & $\%$ \\
\hline \multicolumn{7}{|c|}{ Ocupacional (AFO) } \\
\hline AT & 52 & 56,5 & 25 & 27,2 & 48 & 52,2 \\
\hline SED & 62 & 62,0 & 32 & 32,0 & 51 & 51,0 \\
\hline$* T O(\mathrm{IC}-95 \%)$ & \multicolumn{2}{|c|}{$0,77(0,42-1,42)$} & \multicolumn{2}{|c|}{$0,87(0,44-1,69)$} & \multicolumn{2}{|c|}{$0,99(0,53-1,84)$} \\
\hline \multicolumn{7}{|c|}{ Tempo Livre (AFTL) } \\
\hline AT & 49 & 72,1 & 26 & 38,2 & 31 & 45,6 \\
\hline SED & 65 & 54,2 & 31 & 25,0 & 68 & 54,8 \\
\hline$* T O(\mathrm{IC}-95 \%)$ & \multicolumn{2}{|c|}{$2,36(1,18-4,70)$} & \multicolumn{2}{|c|}{$1,70(0,84-3,41)$} & \multicolumn{2}{|c|}{$0,84(0,43-1,64)$} \\
\hline \multicolumn{7}{|l|}{ Locomoção (AFL) } \\
\hline AT & 47 & 63,5 & 20 & 27,0 & 39 & 52,7 \\
\hline SED & 67 & 56,8 & 37 & 31,4 & 60 & 50,8 \\
\hline$* T O(\mathrm{IC}-95 \%)$ & \multicolumn{2}{|c|}{$1,48(0,78-2,80)$} & \multicolumn{2}{|c|}{$0,88(0,44-1,74)$} & \multicolumn{2}{|c|}{$0,99(0,52-1,86)$} \\
\hline \multicolumn{7}{|l|}{ AF total } \\
\hline AT & 60 & 71,4 & 28 & 33,3 & 42 & 50,0 \\
\hline SED & 54 & 50,0 & 29 & 26,9 & 57 & 52,8 \\
\hline$* T O(\mathrm{IC}-95 \%)$ & \multicolumn{2}{|c|}{$2,50(1,33-4,72)$} & \multicolumn{2}{|c|}{$1,40(0,72-2,69)$} & \multicolumn{2}{|c|}{$0,94(0,50-1,74)$} \\
\hline
\end{tabular}

Para uma análise mais detalhada sobre a influência dos fatores de risco (sexo, etnia, escolaridade, tabagismo, hábito alcoólico e circunferência de cintura) sobre a presença de demais patologias, a casuística foi agrupada segundo o número de fatores de risco presentes (entre 1 e 2 fatores; entre 3 e 4; e mais de 5 fatores de risco), e ainda, dividida entre as 
naturezas de atividade física segundo a atividade predominantemente exercida (Tabela 8). O único grupo que apresentou diferenças significantes foi o de AFO para doenças endócrino-metabólicas, como pode ser observado na tabela 8.

Tabela 8. Distribuição da casuística segundo número de fatores de risco em relação à ausência de doenças, nível e categoria de atividade física.

\begin{tabular}{|c|c|c|c|c|c|c|}
\hline \multirow[b]{3}{*}{ Atividade Física } & \multicolumn{6}{|c|}{ Classificação Internacional de Doenças - CID } \\
\hline & \multicolumn{2}{|c|}{$\mathrm{E} 00-\mathrm{E} 90$} & \multicolumn{2}{|c|}{$\mathrm{I} 00-\mathrm{I} 99$} & \multicolumn{2}{|c|}{ M00 - M99 } \\
\hline & Presente & Ausente & Presente & Ausente & Presente & Ausente \\
\hline \multicolumn{7}{|l|}{ Ocupacional } \\
\hline $1|--| 2$ & 34 & 18 & 10 & 42 & 30 & 22 \\
\hline $3|--| 4$ & 30 & 30 & 17 & 43 & 36 & 24 \\
\hline$>5$ & 03 & 06 & 04 & 05 & 03 & 06 \\
\hline$\chi^{2}(\mathrm{P}=)$ & \multicolumn{2}{|c|}{$4,58(0,03)$} & \multicolumn{2}{|c|}{$3,01(0,09)$} & \multicolumn{2}{|c|}{$2,88(0,46)$} \\
\hline \multicolumn{7}{|l|}{ Tempo Livre } \\
\hline $1|--| 2$ & 13 & 06 & 07 & 12 & 06 & 13 \\
\hline $3|--| 4$ & 19 & 08 & 09 & 18 & 13 & 14 \\
\hline$>5$ & 02 & 01 & 02 & 01 & 01 & 02 \\
\hline$\chi^{2}(\mathrm{P}=)$ & \multicolumn{2}{|c|}{$0,03(0,95)$} & \multicolumn{2}{|c|}{$1,29(0,66)$} & \multicolumn{2}{|c|}{$1,43(0,45)$} \\
\hline \multicolumn{7}{|l|}{ Locomoção } \\
\hline $1|--| 2$ & 02 & 00 & 01 & 01 & 01 & 01 \\
\hline $3|--| 4$ & 05 & 07 & 04 & 08 & 06 & 06 \\
\hline$>5$ & - & - & - & - & - & - \\
\hline
\end{tabular}

Nota: Teste Qui-quadrado $\left(\chi^{2}\right)$. Classificação Internacional de Doenças $(C I D)=$ CID E00-E90: Doenças endócrinas, nutricionais e metabólicas; CID I00-I99: Doenças do aparelho circulatório; CID M00-M99: Doenças do sistema osteomuscular e do tecido conjuntivo.

Para melhor visualização da associação entre os níveis de atividade física nas diferentes categorias estudadas com os valores de pressão arterial sistólica e diastólica, foi realizada análise de regressão linear e de correlação em três estas variáveis. A Ilustração 1 apresenta os gráficos correspondentes às análises para cada categoria de atividade física e pressão arterial sistólica. Observa-se relação inversa entre PAS e atividade de locomoção (gráfico C) e atividade física total (gráfico D), com valores de correlação significantes ( $\mathrm{p}=$ 0,02 e 0,008 , respectivamente). 


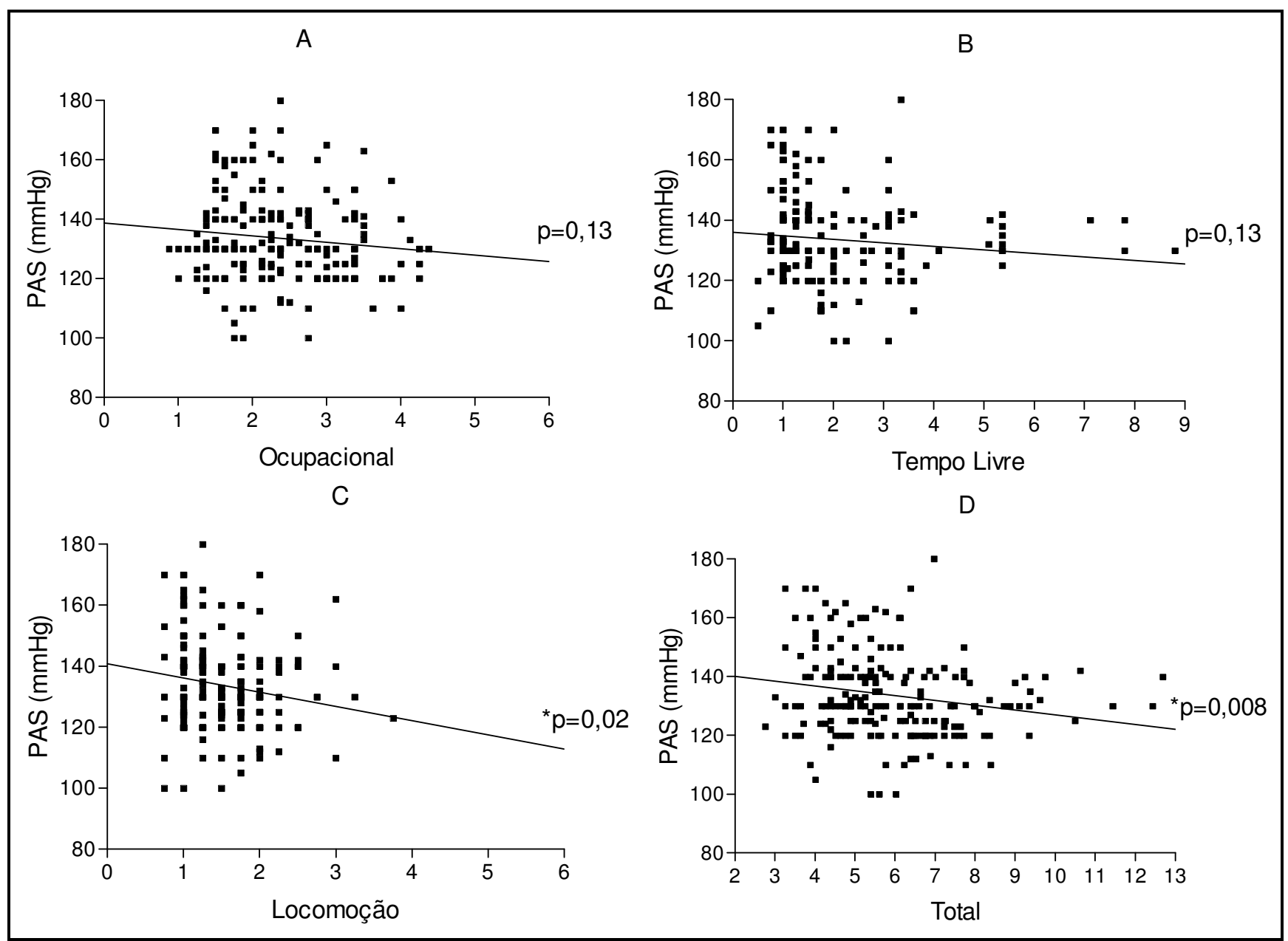

Ilustração 1. Regressão linear entre pressão arterial sistólica (PAS) e categorias de atividade física, com respectivos valores de significância (p) para a correlação entre as variáveis. *= correlação de Pearson.

Na Ilustração 2 são apresentadas as mesmas análises, para os valores de pressão arterial diastólica. Correlação estatística significante não foi observada para nenhuma das categorias de atividade física. Percebe-se a mais influente sobre os valores de 
PAD a AFL, mostrando relação inversa entre as variáveis.
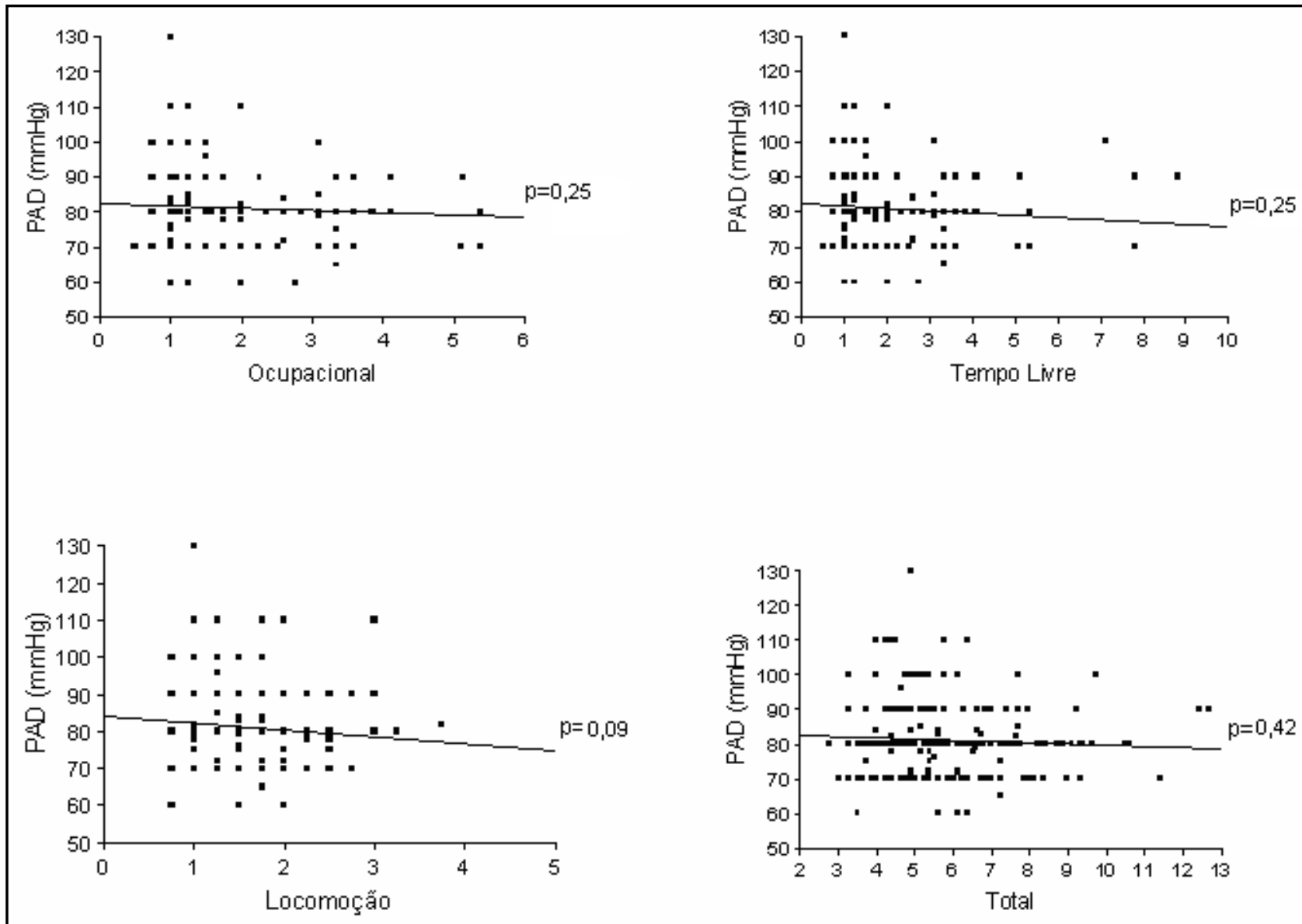

Ilustração 2. Regressão linear entre valores de pressão arterial diastólica (PAD) e escores de atividade física, com respectivos valores de significância (p) para a correlação entre as variáveis. *= correlação de Pearson.

\section{DISCUSSÃO}

Perfil da casuística: associações entre fatores de risco.

A casuística composta por 192 pacientes hipertensos observada no presente trabalho, é na sua maioria pertencente à etnia branca, tem escolaridade $<8$ anos de estudos, e média de idade de 63 anos. Embora todos sejam diagnosticados com HAS, possivelmente pelo uso de medicamentos e controle da alimentação com apoio de nutricionista 
disponibilizada pelo núcleo de saúde, a média de PAS e PAD encontra-se abaixo dos valores estabelecidos pela V DBHA ${ }^{(1)}$ que caracterizam a hipertensão.

A prevalência de pacientes brancos foi $61,5 \%$ seguidos dos negros com $30,7 \%$. Contrariamente, em trabalho publicado por Stevens et al., ${ }^{(109)}$ a prevalência de HAS foi maior entre os negros, comparado aos participantes brancos $(44,2 \%$ vs $22,5 \%)$.

Em relação ao nível de atividade física, o escore médio de AF Total foi 5,92 \pm 1,76, sendo a AFO a categoria com maior escore do grupo como um todo $(2,37 \pm 0,77)$ e a AFL como a de menor escore $(1,55 \pm 0,54)$. Após divisão entre os grupos ativo (AT) e sedentário (SED), a maior média observada para o grupo AT foi a AFTL $(3,48 \pm 1,50)$ e a menor foi a AFL $(2,10 \pm 0,42)$, enquanto que no grupo SED a maior média foi observada para a AFO $(1,76 \pm 0,33)$ e as menores foram apresentadas para AFTL e AFL, ambas com escore de 1,20 de média.

Em levantamento realizado com 192 idosos $(70,7 \pm 7$ anos) para identificação do NAF e doenças crônicas, os escores de atividade física foram mais baixos que os obtidos na presente pesquisa, usando-se mesmo inquérito (modificado para idosos) empregado neste trabalho, apresentando média de 3,5 para a AF Total, 1,36 e 1,80 para AFO de homens e mulheres, respectivamente, e 0,11 e 0,005 para AFTL, homens e mulheres, respectivamente (110)

Tais diferenças nos resultados podem ser explicadas pela característica da casuística deste grupo, com idade mais avança que o observado na presente pesquisa, e por se tratar predominantemente por idosos aposentados. 
Nível de atividade física e fatores de risco

Em relação ao tabagismo como fator de risco para patologias, na presente pesquisa não houve relação de risco ou proteção quando consideradas as doenças do sistema circulatório $(\mathrm{OR}=1,84 ; \mathrm{IC}=0,70-4,85)$, doenças metabólicas $(\mathrm{OR}=0,46 ; \mathrm{IC}=0,18-1,20)$ e do sistema osteomuscular $(\mathrm{OR}=0,66 ;(0,25-1,71)$. Nenhuma destas associações obteve significância estatística relevante. Resultados de Santos, Ebrahim e Barros (112) não encontraram associações do uso de tabaco com síndrome metabólica entre homens e mulheres, dados estes que contrariam os dados de Shankar et al., ${ }^{(113)}$.

Considerando a atividade física, no estudo de Abete et al., ${ }^{(14)}$ houve diminuição do número de fumantes com o aumento do nível de atividade física, e correspondente diminuição dos casos de HAS, mas sem diferenças estatísticas. Neste mesmo trabalho foi observada redução da incidência de óbitos com aumento do NAF (p<0,001). Corroborando com esses dados, Manini et al., ${ }^{(115)}$ não observaram diferenças significantes no hábito de fumar com aumento do nível de atividade física. Tais dados contrariam a expectativa de que indivíduos fisicamente ativos tenham maior consciência sobre os elementos determinantes de saúde, controlando os hábitos negativos do estilo de vida. Porém, para o hábito de fumar, pode-se esperar que seja um comportamento de difícil controle voluntário, por se tratar de hábito que causa dependência química.

Nível de atividade física e indicadores de saúde

O ponto de corte para caracterização de risco coronariano segundo a circunferência de cintura (CC) utilizado na presente pesquisa também foi adotado em estudos prévios, com associações positivas para a HAS e demais morbidades ${ }^{(116-118)}$. As médias de IMC e CC 
da casuística revelam prevalência de sobrepeso $(\mathrm{IMC}=29,78 \pm 6,53$ para mulheres e 29,70 $\pm 6,26$ para homens $)$ e risco cardiovascular $(\mathrm{CC}=96,79 \mathrm{~cm} \pm 13,55 \mathrm{~cm}$ para mulheres e $104,22 \mathrm{~cm} \pm 20,24 \mathrm{~cm}$ para homens), quando considerados ambos os indicadores, que são classicamente relacionados à HAS e demais doenças crônicas.

O nível de atividade física nas diferentes categorias estudadas, não mostrou associação com estes indicadores antropométricos entre ativos e sedentários. Em recente estudo conduzido por Heitmann et al., ${ }^{(119)}$ diferenças significantes entre ativos e sedentários foram observadas em relação ao peso corporal (p<0,05), IMC (p<0,01), e CC (p<0,001). Neste estudo com follow up de 13 anos, as taxas de mortalidade associaram-se ao IMC elevado e inatividade física, onde $27 \%$ dos inativos e $13 \%$ dos ativos morreram neste período. Nossos dados não respondem relação de causa e efeito tratando-se destas variáveis por se tratar de corte transversal, sendo assim, sem possibilitar relações com a pressão arterial nestes indivíduos. Contrariamente, examinando a relação entre redução de peso e consequiências sobre a PA, no trabalho de Neter et al., ${ }^{(120)}$, a redução de $5,1 \mathrm{Kg}$ no peso corporal foi responsável pela queda de $4,44 \mathrm{mmHg}$ na PAS e $3,57 \mathrm{mmHg}$ na PAD. Em média a PAS caiu em $1,05 \mathrm{mmHg}$ e a PAD em $0,92 \mathrm{mmHg}$ por $\mathrm{Kg}$ de peso corporal reduzido. Neste mesmo trabalho, as reduções da PAS/ PAD foram mais pronunciadas com uso de medicação anti-hipertensiva.

Embora se espere que maiores médias de atividade física e esportes no tempo livre possam significar diferenças sobre variáveis antropométricas que refletem maiores taxas de gordura corporal, a presente pesquisa não exibe diferenças entre ativos e sedentários quando considerada a AFTL, em relação ao peso e IMC. Ambos os grupos são semelhantes mesmo com escores distintos nesta categoria de atividade física. No estudo de Kronenberg et al., ${ }^{(121)}$ atividades sedentárias no tempo livre, como assistir televisão, foram responsáveis 
pelos aumentos nos valores de IMC e dobras cutâneas, representando fator de risco para sobrepeso entre homens $(\mathrm{RR}=2,12 ; \mathrm{IC}=1,45-3,10)$ e mulheres $(\mathrm{RR}=1,61 ; \mathrm{IC}=1,07$ 2,43). Tais resultados permitem que se façam recomendações de aumento do gasto energético por meio desse tipo de atividade. Neste grupo, possivelmente as médias do escore nesta categoria ainda foram baixas, mesmo para os ativos, sendo assim, as associações com indicadores de saúde (IMC, CC, PAS e PAD) não puderam ser observadas.

Trabalho de Poiorier et al., ${ }^{(122)}$ analisando associação de indicadores antropométricos CC e IMC com PA em 1944 homens e mulheres, observou correlações significantes entre estas variáveis $(\mathrm{p}<0,001)$, sendo que para as mulheres os valores de correlação apresentaram-se sempre mais elevados. Os autores indicaram que homens com IMC normal, porém com $\mathrm{CC}>88 \mathrm{~cm}$ exibiam valores mais elevados de PAS, comparados com aqueles com CC normal $(<88 \mathrm{~cm})$. Trabalho de Stevens ${ }^{(109)}$ demonstrou que valores de IMC elevados significaram aumento correspondente da PA, em todos os grupos étnicos estudados.

Contrariamente, nossos resultados não demonstram associação dos valores aumentados de CC e sedentarismo nas diferentes categorias. Apenas uma inversão interessante é observada para a AFO, onde a maioria dos indivíduos com CC normal (<102/ $88 \mathrm{~cm}$ ) foi classificada como ativa para esta categoria de atividade física $(55,1 \%)$, e contrariamente, a maioria daqueles que apresentaram valores acima do ponto de corte é considerada sedentária $(54,5 \%)$. 
Nível de atividade física e comorbidades

Resultados desta pesquisa associam negativamente, maior nível de atividade física com doenças metabólica, endócrinas e nutricionais no caso da atividade física total (2,50; $\mathrm{IC}=1,33$ - 4,72) e de tempo livre $(2,36$; $\mathrm{IC}=1.18-4,70)$, sem exibir relação de risco ou proteção comparando-se ativos e sedentários para as demais categorias de atividade física com doenças cardiovasculares e osteoarticulares. Pesquisa de Abete et al., ${ }^{(14)}$ realizada com 557 adultos com idade superior a 65 anos, não constatou associação entre nível de atividade física e presença de angina. Contrariamente, o follow up de 12 anos realizado por Franco et al., ${ }^{(123)}$ observou que a atividade física representou fator de proteção para DCV. Houve também, concomitante ao aumento do nível de atividade física, a tendência de redução de colesterol, pressão arterial e taxas de comorbidades entre os 2336 participantes da pesquisa. Devido ao fato do estudo ser transversal, não permitindo inferir relações de causa e efeito entre os desfechos observados, porém, estima-se que por se tratar de grupo em tratamento ambulatorial, não somente para HAS, mas também outras patologias, que estes pacientes tenham procurado aumentar os níveis de atividade física por recomendação médica, após o diagnóstico da doença.

O estudo de Manini et al., ${ }^{(115)}$ não encontrou diferenças relacionadas à presença/ ausência de doenças crônicas (câncer, diabetes, osteoartrite e osteoporose) associadas ao nível de atividade física. No entanto, observaram redução na taxa de mortalidade em indivíduos com maior gasto energético. Os autores sugerem que qualquer atividade física, e não apenas o exercício físico, pode colaborar para a redução das taxas de mortalidade na população. 
Hu et al., ${ }^{(124)}$ examinando associação entre atividade física e IMC com risco de mortes em estudo de coorte de aproximadamente 18 anos, identificaram níveis de atividade física mais elevados como fator de proteção para mortalidade por todas as causas e por DCV, tanto em homens como em mulheres. O IMC $>30 \mathrm{Kg} / \mathrm{m}^{2}$ representou fator de risco, também para mortalidade por todas as causas e por DCV. Indivíduos inativos obesos apresentaram taxa de risco de 1,78 e 2,10 entre homens e mulheres, respectivamente, para mortalidade por todas as causas, comparado aos sujeitos ativos eutróficos. Em relação às doenças cardiovasculares, as taxas foram de 2,09 para homens, e 2,18 para mulheres.

Investigação recente de Steptoe e McMunn ${ }^{(40),}$ avaliando associação de fatores de risco e atividade física com comorbidades, encontrou maior prevalência de DCV, diabetes, infarto e artrite entre hipertensos, quando comparados a normotensos $(\mathrm{p}<0,001$, ajustado por sexo e idade). Em relação à atividade física, o sedentarismo representou fator de risco para comorbidades e atividade física vigorosa foi fator de proteção.

No estudo de Santos, Ebrahim e Barros ${ }^{(112)}$ o aumento do nível de atividade física exibiu tendência de proteção contra síndrome metabólica, tanto para atividade física total, como para a ocupacional (trabalhos domésticos) e de tempo livre. Corroborando com esses dados, Halldin et al., ${ }^{(118)}$ em estudo longitudinal com 4228 homens e mulheres, observaram aumento do nível de atividade física no tempo livre com redução da incidência de síndrome metabólica. Nesta investigação, a AFO não se associou com a incidência da doença.

Mattheus et al., ${ }^{(125)}$ estudando o nível de atividade física para a locomoção considerando caminhada e percursos de bicicleta, e atividade ocupacionais (em casa), constataram a redução da taxa de mortalidade por todas as causas e por doenças cardiovasculares em razão direta ao estilo de vida mais ativo, sendo que, apenas a caminhada apresentou valores estatísticos significantes. Comparando inativos e ativos, e os 
percursos de bicicleta demonstraram apenas tendência de proteção em relação à mortalidade precoce.

Todos estes dados colocam a atividade física em lugar de destaque em relação ao efeito protetor que exerce sobre o organismo tratando-se de patologias diversas. Principalmente nas investigações longitudinais é possível estabelecer relações de proteção as médias mais elevadas de esforço físico. O que não pode ser percebido pelos nossos achados. Vale lembrar que trata-se de corte transversal, e talvez esta seja a explicação para a falta de associações deste tipo. Muito possivelmente os indivíduos participantes desta pesquisa adoeceram antes de aumentarem o nível de atividade física, nas diversas categorias.

Indicadores de saúde e comorbidades

Nossos resultados não apresentaram relação de risco para a CC maior que $102 \mathrm{~cm}$ para homens e $88 \mathrm{~cm}$ para mulheres com outras doenças. Para doenças metabólicas e endócrinas existe tendência de risco com aumento da CC $(1,77 ; \mathrm{IC}=0,92-3,40)$, assim como para doenças do sistema circulatório $(1,64 ; \mathrm{IC}=0,77-3,49)$. Contrariamente, para doenças osteoarticulares foi observada tendência de proteção para CC com valores abaixo de $102 / 88 \mathrm{~cm}$ para homens e mulheres, respectivamente $(0,83 ; \mathrm{IC}=0,43-1,59)$.

Onat et al., ${ }^{(117)}$ verificaram valores mais elevados de CC entre indivíduos com síndrome metabólica e, risco aumentado de desenvolver diabetes entre homens e mulheres entre os portadores de síndrome metabólica. Neste estudo, os valores de pressão arterial sistólica e diastólica também sofreram aumentos concomitantes com obesidade (131/85mmHg para homens e 131/83 para mulheres) e presença da síndrome metabólica 
(140/90mmHg para homens e 152/93mmHg para mulheres), comparados aos indivíduos com peso normal sem a doença $(120 / 77 \mathrm{mmHg}$ para homens e $120 / 77 \mathrm{mmHg}$ para mulheres). No estudo de Olinto et al., ${ }^{(11)}$ foi observado risco para HAS quando a circunferência de cintura foi maior que $80 \mathrm{~cm}(\mathrm{OR}=6,21$; IC=4,30 - 8,98) e o $\mathrm{IMC}>30$ $\mathrm{Kg} / \mathrm{m}^{2}(\mathrm{OR}=6,94 ; \mathrm{IC}=4,80-10,04)$. Para o diabetes, o risco relacionado à $\mathrm{CC}$ foi de 5,42 $(2,57-11,46)$ e de $8,53(4,30-16,52)$ relacionado ao $\mathrm{IMC}>30 \mathrm{Kg} / \mathrm{m}^{2}$.

Em relação às doenças osteomusculares e NAF, nossos resultados não apresentam significância estatística entre estas associações. Nesta direção, Feskanichi, Willet e Colditz (126) observaram que a AFTL de mulheres na pós menopausa foi considerada como fator de proteção para fraturas $(\mathrm{OR}=0,45 ; \mathrm{IC}=0,32-0,63)$, o risco de fratura diminui em $6 \%$ para cada aumento de 3MET no nível de atividade física.

Consumo alcoólico e tabagismo vs HAS e comorbidades;

O presente estudo não encontrou associação do uso de tabaco e consumo alcoólico com comorbidades associadas à hipertensão arterial. Resultados com significância estatística foram observados apenas em relação às doenças osteomusculares, onde foi verificado fator de proteção entre os pacientes que não consomem álcool regularmente $(0,33 ; \mathrm{IC}=0,11-0,96)$

Em estudo longitudinal de cinco anos, Shankar et al., ${ }^{(113)}$, constataram que o baixo nível educacional, hábito de fumar e IMC $>30 \mathrm{Kg} / \mathrm{m}^{2}$, mostraram-se associados. Contrariamente, o consumo de álcool não exibiu a mesma relação com prevalência de doenças. Neste estudo, a HAS quando presente apresentou risco de 5,79 (IC 95\%=4,41 7,61) para outras doenças. Santos, Ebrahim e Barros ${ }^{(112)}$ observaram aumento da 
prevalência de síndrome metabólica com aumento do consumo de álcool, em pesquisa realizada em Portugal com 2164 homens e mulheres.

As limitações do presente trabalho podem ser descritas pelo uso de questionário para identificação do nível de atividade física, sem critério estabelecido como ponto de corte para divisão entre grupos de ativos e sedentários, porém, em estudos com grandes populações este instrumento é considerado válido, pelo baixo custo e facilidade de aplicação. Os dados foram coletados em núcleos de saúde, o que faz da casuística um caso especial, onde todos pacientes já apresentavam alguma enfermidade, além da hipertensão arterial diagnosticada.

\section{CONCLUSÕES}

As variáveis antropométricas IMC, CC e peso corporal total não são influenciadas pela categoria de atividade física, comparando-se pacientes considerados sedentários e ativos em cada uma delas. Valores de pressão arterial de repouso parecem ser influenciados pelas atividades AFTL, AFL e AF total, sempre os menores valores médios foram observados no grupo sedentário.

A ocorrência de comorbidades também não parece ser determinada pelo nível de atividade física, pois, somente em duas categorias de AF (AFTL e AF total) foram observados resultados significantes sobre doenças endócrino-metabólicas, porém, em ambas o comportamento ativo representou fator de risco para ocorrência de doenças destas categorias. Demais grupos de doenças não apresentaram diferenças entre ativos e sedentários em nenhuma categoria de AF. 


\section{REFERÊNCIAS}

1- V DIRETRIZES BRASILEIRAS DE HIPERTENSÃO ARTERIAL. Hipertensão. Sociedade Brasileira de Hipertensão. Revista Hipertensão 2006; 9 (4).

2- Williams, PT. A cohort study of incident hypertension in relation to changes in vigorous physical activity in men and women. J Hypertens 2008; 26(6):1085-1093.

3- El-Atat, FA; Stas, SN; Mcfarlane, SI; Sowers, JR. The relationship between hyperinsulinemia, hypertension and progressive renal disease. Journal of the American Society oh Nephology 2004; 15(11): 2816-1827.

4- Blair, SN; Kohl, HW; Gordon, NF. How much physical activity is good for health? Annu Rev Public Health 1992; 13: 99-126.

5- Conceição, TV; Gomes, FA; Tauil, PL; Rosa, TT. Valores de pressão arterial e suas associações com fatores de risco cardiovasculares em servidores da Universidade de Brasília. Arq Bras Cardiologia 2006; 86(1):26 - 31.

6- Fagard, RH. Exercise characteristics and the blood pressure response to dynamic physical training. Med Sci Sports Exercise 2001; 33(6): S484-S492 Je.

7- Stewart, KJ. Exercise training and the cardiovascular consequences of type 2 diabetes and hypertension. JAMA 2003; 288(13): 1622-1631.

8- Monteiro, HL; Rolim, LMC; Squinca, DA; Silva, FC; Ticianeli, CCC; Amaral, SL. Efetividade de um programa de exercícios no condicionamento físico, perfil metabólico e pressão arterial de pacientes hipertensos. Rev Bras Med Esporte 2007; 13(2): 107-112.

9- Kokkinos, P; Pittaras, A; Manolis, A; Panagiotakos, D; Narayan, P; Manjoros, D; et al.Exercise capacity and 24-h blood pressure in prehypertensive men and women. Am J Hypertension 2006; 19(3): 251-258.

10- Rondon, MUPB; Brum, PC. Exercício físico como tratamento não farmacológico da hipertensão arterial. Rev Bras Hipertensão 2003; 10: 134-139.

11- Hu, G; Tuomilehto, J; Borodulin, K; Jousilahti, P. The joint associations of occupational, commuting, and leisure-time physical activity, and the Framingham risk score on the 10-year risk of coronary heart disease. European Heart Journal 2007; 28: 492498.

12- Tanascescu, M; Leitzmann, MF; Rimm, EB; Willett, WC; Stampfer, MJ; Hu, FB. Exercise type and intensity in relation to coronary heart disease in men. Jama 2002; 288(16): $1994-2000$ 
13- AMERICAN COLLEGE OF SPORTS MEDICINE. Physical Activity and Public Health: Updated Recommendation for Adults from the American College of Sports Medicine and the American Heart Association. Med Sci Sports Exercise 2007; 39(8):14231434.

14- Barengo, NC; Hu, G; Lakka, TA; Pekkarinen, H; Nissinen, A; Tuomilehto, J. Low physical activity as a predictor for total and cardiovascular disease mortality in middle-aged men and women in Finland. European Heart Journal 2004; 25(24): 2204-2211.

15- Hu, G; Barengo, NC; Tuomilehto, J; Lakka, TA; Nissinen, A; Jousilahti, P. Relationship of physical activity and Body Mass Index to the risk of hypertension: a prospective estudy in Finland. Hypertension 2004; 43(1):25-30.

16- Costa, JSD; Barcellos, FC; Sclowitz, ML; Sclowitz, IKT; Castanheira, M; Olinto, MTA. et al. Hypertension prevalence and its associated risk factors in adults: a populationbased study in Pelotas. Arq Bras Cardiol 2007; 8(1): 54-59.

17- Fagard, RH. Physical activity, physical fitness and incidence of hypertension. Journal of Hypertension 2005; 23(2): 265-267.

18- Monteiro, CA; Conde, WL; Matsudo, SM; Matsudo, VR; Bonseñor, I M; Lotufo, PA. A descritive epidemiology of leisure-time physical active in Brazil, 1996-1997. Panamerican Journal of Public Health 2003; 14(4): 246 - 254

19- Matsudo, SM; Matsudo, VR; Araújo, T; Andrade, D; Andrade, E; Oliveira, L. et al. Nível de atividade física da população do estado de São Paulo: análise de acordo com gênero, idade, nível sócio econômico, distribuição geográfica e de conhecimento. Rev Bras Ciên e Mov 2002; 10(4): 41-50.

20- Riddoch, CJ; Andersen, LB; Wedderkopp, N; Harro, M; Klasson-Heggebo, L; Sardinha, LB; et al. Physical Activity Levels and Patterns of 9- and 15-yr-Old European Children. Med Sci Sports Exercise 2004; 6(1): 86-92.

21- Hootman, JM; Macera, CA; Ham, SA; Helmick, CG; Sniezek, JE. Physical activity levels among the general US adult population and in adults with and without arthritis. Arthritis \& Rheumatism 2003; 49(1): 129-135.

22- Jakicic, JM; Otto, AD. Physical activity considerations for the treatment and prevention of obesity. Am J Clin Nutr 2005; 82(1) 226S-229S.

23- Hallal, PC; Victoria, CG; Wells, JCK; Lima, RC. Physical inactivity: prevalence and associated variables in brazilian adults. Med. Sci. Sports Exerc 2003; 35(11): 1894-1900. 
24- Ross, R. Jansen, I. Physical activity, total and regional obesity: dose-response considerations. Med Sci Sports Exercise 2001; 33(6 Suppl): S521-S527.

25- Chobanian, AM; Bakris, GL; Black, HR; Cushman WC; Green, LA; Izzo, JL; et al. Seventh report of the Joint National Committee on prevention, detection, evaluation, and treatment of high blood pressure. Hypertension 2003; 42(6): 1206-1252.

26- Weinstein, AR; Sesso, HD; Lee, M; Cook, NR; Manson, JE; Buring, JE; et al. Relationship of physical activity vs Body Mass Index with type 2 diabetes in women. JAMA 2004; 292(10): 1188-1194.

27- Guedes, DP; Gonçalves, LAVV. Impacto da Prática Habitual de Atividade Física no Perfil Lipídico de Adultos. Arq Bras Endocrinol Metab 2007; 51(1):72-78.

28- Bassuk, SS; Manson, JE. Epidemiological evidence for the role of physical activity in reducing risk of type 2 diabetes and cardiovascular disease. J Appl Physiol 2005; 99(3): 1193-1204.

29- Yu, S; Yarnell, JWG; Sweetnam, PM; Murray, L. What level of physical activity protects against premature cardiovascular health? The Caerphilly study. Heart 2003; 89(5):502-506.

30- Laaksonen, DE; Lakka, HM; Salonen, JT; Niskanen, LK; Rauramma, R; Lakka, TA. Low levels of leisure-time physical activity and cardiorespiratory fitness predict development of the metabolic syndrome. Diabetes Care 2002; 25(9):1612-1618.

31- Gu, D; Wildman, RP; Wu, X; Reynolds, K; Huang, J; Chen, CS; He, J. Incidence and predictors of hypertension over 8 years among Chinese men and women. J Hypertens 2007; 25(3): 517-523

32- Andersen, LB; Schnohr, P; Schroll, M; Hein, HO. All-Cause Mortality Associated With PhysicalActivity During Leisure Time, Work, Sports, and Cycling to Work. Arch Intern Med 2000;160(11): 1621-1628

33- Passos, VMA; Assis, TD; Barreto, SM. Hipertensão arterial no Brasil: estimativa de prevalência a partir de estudos de base populacional. Epidemiologia e Serviços de Saúde 2006; 15(1): 35-45.

34- Sanchez, RA; Ayala, M; Baglivo, H; Velazquez, C; Burlando, G; Kohlmann, O; et al. Latin American guidelines on hypertension. J Hypertens 2009, 27(5):905-922.

35- Xin, X; He, J; Frontini, MG; Ogden, LG; Motsamai, OI; Whelton, PK. Effects of alcohol reduction on blood pressure: a meta-analysis of randomized controlled trials. Hypertension. 2001; 38(5): 1112-1117. 
36- Whelton, PK; He, J; Appel, LJ; Cutler, JA; Havas, S; Kotchen, TA. et al. Primary Prevention of Hypertension. JAMA. 2002; 288(15): 1882-1888

37- Appel, et al. Effects of Comprehensive Lifestyle Modification on Blood Pressure Control. Main Results of the PREMIER Clinical Trial. JAMA, 2003; 289(16): 2083 - 2093.

38- Hall, JE; Granger, JP; Reckelhoff, JF; Sandberg, K. Hypertension and Cardiovascular Disease in Women. Hypertension 2008;51;951.

39- Thomas, F; Rudnichi, A; Bacri, A; Bean, K; Guize, L; Beneto, A. Cardiovascular Mortality in Hypertensive Men According to Presence of Associated Risk Factors. Hypertension 2001; 37(5): 1256-1261.

40- Steptoe, A; Mcmunn, A. Health behaviour patterns in relation to hypertension: the English Longitudinal Study of Ageing. J Hypertens 2009; 27(2): 224-230.

41- Vasan, RS; Larson, MG; Leip, EP; Evans, JC; O`Donnel, CJ; Kannel, WB; Levy, D. Impact of high-normal blood pressure on the risk of cardiovascular disease. N Engl J Med 2001; 345(18): 1291-7.

42- Greenberg, J. Are Blood Pressure Predictors of Cardiovascular Disease Mortality Different for Prehypertensives Than for Hypertensives? Am J Hypertens 2006; 19(5): 454461.

43- Brown, DW; Giles, WH; Greelund, KJ. Blood Pressure Parameters and Risk of Fatal Stroke. NHANES II Mortality Study. Am J Hypertens 2007; 20(3): 338-341.

44- Weycker, D; Nichols, GA; O’Keeffe-Rosetti, M; Edelsberg, J; Khan, ZM; Kaura, S; Oster, G. Risk-Factor Clustering and Cardiovascular Disease Risk in Hypertensive Patients. Am J Hypertens 2007; 20(6):599-607

45- Kannel, WB. Risk stratification in hypertension: new insights from the Framingham study. Am J Hypertension, 2000;13: 3S-10S.

46- Lopes, PP; Vanderlei, LCM; Tarumoto, MH. Perfil dos indivíduos frequentadores de programas ambulatoriais de exercícios físicos para pacientes cardíacos da cidade de Presidente Prudente - SP. Revista da Sociedade de Cardiologia do Estado de São Paulo 2005; 15(3): (supl A) 19-28.

47- Chei, C, Isso, H; Yamagishi, K; Tanigawa, T; Cui, R; Imano, H; Kiyama, M; Kitamura, A; Sato, S; Shimamoto, T. Body Fat Distribution and the Risk of Hypertension and Diabetes among Japanese Men and Women. Hypertens Res 2008; 31(5): 851-857.

48- Kotsis, V; Stabouli, S; Bouldin, M; Low, A; Toumanidis, S; Zakopoulos, N. Impact of Obesity on 24-Hour Ambulatory Blood Pressure and Hypertension. Hypertension. 2005; 45(4): 602-607. 
49- Fuchs, FD; Gus, M; Moreira, LB; Moraes, RS; Wiehe, M; Pereira, GM; Fuchs, SC. Anthropometric Indices and the Incidence of Hypertension: A Comparative Analysis. Obes Res. 2005; 13(9): 1515-1517.

50- Gus, M; Fuchs, SC; Moreira, LB; Moraes, RS; Wiehe, M; Silva, AF; Albers, F; Fuchs, FD. Association Between Different Measurements of Obesity and the Incidence of Hypertension. Am J Hypertens 2004; 17(1):50-53.

51- Niskanen, L; Laaksonen, DE; Nyyssönen, K; Punnonen, K; Valkonen, V; Fuentes, R; Tuomainen, T; Salonen, R; Salonen, JT. Inflammation, Abdominal Obesity, and Smoking as Predictors of Hypertension. Hypertension 2004; 44(6): 859-865.

52- Blumenthal, JA; Sherwood, A; Gullette, ECD; Babyak, M; Waugh, R; Georgiades, A; et al. Exercise and Weight Loss Reduce Blood Pressure in Men and Women With Mild Hypertension. Arch Intern Med. 2000; 160(13):1947-1958.

53- Tsai, JC; Liu, JC; Kao, CC; Tomlinson, B; Kao, PF; Chen, JW; Chan, P. Beneficial Effects on Blood Pressure and Lipid Profile of Programmed Exercise Training in Subjects With White Coat Hypertension. Am J Hypertens 2002;15(6): 571-576

54- Winnicki, M; Bonso, E; Dorigatti, F; Longo, D; Zaetta, V; Mattarei, M; et al. Effect of Body Weight Loss on Blood Pressure After 6 Years of Follow-Up in Stage 1 Hypertension. Am J Hypertens 2006; 19(11):1103-1109.

55- Myers, J. Exercise and Cardiovascular Health. Circulation 2003;107(1): e2-e5.

56- Schultz, RL; Swallow, JG; Waters, RP; Kuzman, JA; Redetzke, RA; Said, S; De Escobar, GM; Gerdes, AM. Effects of excessive long-term exercise on cardiac function and myocyte remodeling in hypertensive heart failure rats. Hypertension. 2007; 50(2): 410-416.

57- Frey, N; Olson, EN. CARDIAC HYPERTROPHY: The Good, the Bad, and the Ugly. Annu Rev Physiol 2003; 65: 45-79.

58- Caspersen, CJ; Powell, KE; Christenson, GM. Physical Activity, Exercise, and Physical Fitness: Definitions and Distinctions for Health-Related Research. Public Health Reports 1985, 100(2): $126-140$

59- Brum, PC; Forjaz, CLM; Tinucci, T; Negrão, CE. Adaptações agudas e crônicas do exercício físico no sistema cardiovascular. Rev. Paul. Educ. Fís 2004; 18: 21-31.

60- Brasília, 2002. MINISTÉRIO DA SAÚDE. Plano de reorganização da atenção a hipertensão arterial e ao diabetes mellitus. Manual de hipertensão e diabetes mellitus.

61- Paffenbarger, JRS Contribuitions of epidemiology to exercise science and cardiovascular health. Med Sci Sports Exercise 1988; 20(5): 426-438, 1988. 
62- Byberg, L; Zethelius, B; Mckeigue, PM; Lithell, HO. Changes in physical activity are associated with changes in metabolic cardiovascular risk factors. Diabetologia, 2001; 44(12): 2134-2139.

63- Blair, SN; Cheng, Y; Holder, JS. Is physical activity or physical fitness more important in defining health benefits? Med Sci Sports Exercise 2001; 33(6): S379-S399.

64- Roberts, CK; Barnard, RJ. Effects of exercise and diet on chronic disease. J Appl Physiol 2005; 98(1): 3-30.

65- Kraus WE, Houmard JA, Duscha BD, Knetzger KJ, Wharton MB, Mccartney JS. et al. Effects of the amount and intensity of exercise on plasma lipoproteins. N Engl J Med 2002; 347(19): $1483-1492$.

66- Hambrecht R; Adams V; Erbs S; Linke A; Krankel N; Shu Y; Baither Y; Gielen S; Thiele H; Gummert JF; Mohr FW; Schuler G. Regular physical activity improves endothelial function in patients with coronary artery disease by increasing phosphorylation of endothelial nitric oxide synthase. Circulation 2003; 107(25): 3152-3158.

67- Dumortier, M; Brandou, F; Perez-Martin, A; Fedou, C; Mercier, J; Brun, JF. Low intensity endurance exercise targeted for lipid oxidation improves body composition and insulin sensitivity in patients with the metabolic syndrome. Diabetes Metab 2003; 29(5): 509-18.

68- Manson, JE; Greenland, P; Lacroix, AZ; Stefanick, ML; Mouton, CP; Oberman, A; et al. Walking compared with vigorous exercise for the prevention of cardiovascular events in women. N Engl J Med 2002; 347(10): 716-725.

69- Haskell, WL; Lee, IM; Pate, RR; Powell, KE; Blair, SN; Franklin, BA; et al. Physical Activity and Public Health: Updated Recommendation for Adults from the American College of Sports Medicine and the American Heart Association. Med. Sci. Sports Exerc 2007; 39(8): 1423-1434.

70- Pescatello, LS; Franklin, BA; Fagard, R; Farquhar, WB; Kelley, GA; Ray, CA. Exercise and Hypertension. Med Sci Sports Exercise 2004; 36(3): 533 - 553.

71- Thompson, PD; Buchner, D; Piña, IL; Balady, GJ; Williams, MA; Marcus, BH. et al. Exercise and Physical Activity in the Prevention and Treatment of Atherosclerotic Cardiovascular Disease. A Statement From the Council on Clinical Cardiology (Subcommittee on Exercise, Rehabilitation, and Prevention) and the Council on Nutrition, Physical Activity, and Metabolism (Subcommittee on Physical Activity). Circulation 2003; 23(8):e42 - e49.

72- Pate, RR; Pratt, M; Blair, SN; Haskell, WL; Macera, CA; Bouchard, C. et al. Physical activity and public health: a recommendation from the Centers for Disease Control and Prevention and the American College of Sports Medicine. JAMA 1995; 273(5): 402-7 
73- Rolim, LMC; Amaral, SL; Monteiro, HL. Hipertensão e exercício: custos do tratamento ambulatorial, antes e após a adoção da prática regular e orientada de condicionamento físico. Hipertensão 2007; 10: 2-10.

74- Nakanishi, N; Suzuki, K. Daily Life Activity and the Risk of Developing Hypertension in Middle-aged Japanese Men. Arch Intern Med. 2005; 165(2):214-220.

75- Fransson, E; Ahlbom, A; Reuterwall, C; Hallqvist, J; Alfredsson, L. The Risk of Acute Myocardial Infarction: Interactions of Types of Physical Activity. Epidemiology 2004; 15(5): 573-582.

76- Greenlund, KJ; Daviglus, ML; Croft, JB. Differences in healthy lifestyle characteristics between adults with prehypertension and normal blood pressure. J Hypertens 2009, 27(5): 955-962.

77- Swartz, AM; Strath, SJ; Parker, SJ; Miller, NE. The Impact of Body-Mass Index and Steps per Day on Blood Pressure and Fasting Glucose in Older Adults. Journal of Aging and Physical Activity 2008; 16:188-200.

78- Hayashi, TH; Tsumura, K; Suematsu, C; Okada, K; Fujii, S; Endo, G; Walking to work and the risk for hypertension in men: the Osaka health survey. Ann Intern Med. 1999; 130(1):21-26.

79- Wagner, A; Simon, C; Evans, A; Ferrières, J; Montaye, M; Ducimetière, P; Arveiler, D. Physical Activity and Coronary Event Incidence in Northern Ireland and France. Circulation. 2002; 105:2247-2252.

80- Gustat, J; Srinivasan, SR; Elkasabany, A; Berenson, GS. Relation of self-rated measures of physical activity to multiple risk factors of insulin resistance syndrome in young adults: The Bogalusa Heart Study. J Clin Epidemiol 2002; 55(10):997 - 1006.

81- Barengo, NC; Hu, G; Kastarinen, M; Lakka, TA; Pekkarinen, H; Nissinen, A; Tuomilehto, J. Low physical activity as a predictor for antihypertensive drug treatment in 25-64-year-old populations in Eastern and south-western Finland. J Hypertens 2005, 23(2): 293-299.

82- Sobngwi, E; Mbanya, JCN; Unwin, NC; Kengne, AP; Fezeu, L; Minkoulou, AM; et al. Physical activity and its relationship with obesity, hypertension and diabetes in urban and rural Cameroon. Int J Obesity 2002; 26(7): 1009-1016.

83- Oppert, JM; Thomas, F; Charles, MA; BENETOS, A; BASDEVANT, A; SIMON, C. Leisure-time and occupational physical activity in relation to cardiovascular risk factors and eating habits in French adults. Public Health Nutrition 2005; 9(6): 746-754.

84- Apullan, FJ; Bourassa, MG; Tardif, JC; Fortier, A; Gayda, M; Nigam, A. Usefulness of self-reported leisure-time physical activity to predict long-term survival in patients with coronary heart disease. Am J Cardiol 2008; 102(4): 375-379. 
85- Hallal, PC; Dumith, SC; Bastos, JP; Reichert, FF; Siqueira, FV; Azevedo, MR. Evolução da pesquisa epidemiológica em atividade física no Brasil: revisão sistemática. Rev Saúde Pública 2007; 41(3): 453-60.

86- Glaner, MF. Concordância do questionário de atividade física com aptidão cardiorrespiratória. Rev Bras Cineantropometria \& Desempenho Humano 2007; 9(1): 6166.

87- Elmer, PJ; Obarzanek, E; Vollmer, WM; Simons-Morton, D; Stevens, VJ; Young, DR. et al. Effects of Comprehensive Lifestyle Modification on Diet, Weight, Physical Fitness, and Blood Pressure Control: 18-Month Results of a Randomized Trial 2006; Ann Intern Med. 2006. 144 (7): 485-495.

88- Michels, KB; Wolk, A. A prospective study of variety of healthy foods and mortality in women. Int J Epidemiol 2002; 31(4): 847-854.

89- Mccollough, ML; Feskanich, D; Stampfer, MJ; Rosner, BA; Hu, FB; Hunter, DJ. et al. Adherence to the Dietary Guidelines for Americans and risk of major chronic disease in men. Am J Clin Nutr 2000; 72(5): 1223-31.

90- Baecke, JAH, Burema, J; Frijters, JER.A short questionnaire for the measurement of habitual physical activity in epidemiological studies. Am J Clin Nutr 1982; 36: 936-42.

91- Florindo, AA; Latorre, MDRDO. Validation and reliability of the Baecke questionnaire for the evaluation of habitual physical activity in adult men. Rev Bras Med Esporte, 2003. 9(3): 129-135.

92- Guedes, DP; Lopes, CC; Guedes, JERP, Stanganelli, LC. Reprodutibilidade e validade do questionário Baecke para avaliação da atividade física habitual em adolescentes. Rev Port Cien Desp 2006; 6(3): 265-74.

93- Evenson, KR; Rosamond, WD; Cai, J; PEREIRA, MA; Ainsworth, BE. Occupational physical activity in the atherosclerosis risk in communities study. Annals of Epidemiology 2003; 13(5): 351-357.

94- Evenson, KR; Rosamond, WD; Cai, J; Diez-Roux, AV; Brancati, FL. Influence of retirement on leisure-time ahysical activity: the atherosclerosis risk in communities study. Am J Epidemiol 2002; 155(8): 692-699.

95- Krause, MP; Buzzachera, CF; Hallage, T; Pulner, SB; Silva, SG. Influência do nível de atividade física sobre a aptidão cardiorrespiratória em mulheres idosas. Rev Bras Med Esporte 2007; 13(2): 97-102. 
96- Florindo, AA; Latorre, MDRDO; Jaime, PC; Tanaka, T; Zerbini, CA. Methodology to evaluation the habitual physical activity in men aged 50 years or more. Rev Saúde Publica 2004; 38(2): 307-14.

97- Florindo, AA; Latorre, MDRDO; Jaime, PC; Tanaka, T; Pippa, MGB; Zerbini, CAF. Past and Present Habitual Physical Activity and Its Relationship With Bone Mineral Density in Men Aged 50 Years and Older in Brazil. Journal of Gerontology 2002; 57A(10): M654-M657.

98- Hertogh, EM; Monninkhof, EM; Schouten, EG; Peeters, PHM; Schuit, AJ. Validity of the Modified Baecke Questionnaire: comparison with energy expenditure according to the doubly labeled water method. Int J Behav Nutr Phys Act 2008; 5:.30.

99- Pols, MA; Peeters, PHM.; Kemper, HCG; Collette, HJA. Repeatability and relative validity of two physical activity questionnaires in elderly women. Med Sci Sports Exercise 1996; 28(8): 1020-1025.

100- Pols, MA; Peeters, PHM; Bueno-De-Mesquita, HB; Ocke, MC; Wentink, CA; Kemper, HCG; Collette, HJA. Validity and repeatability of a modified Baecke questionnaire on physical activity. Int. J. Epidemiol 1995; 24(2): 381-388.

101- Pastre, CM; Carvalho Filho, G; Monteiro, HJ; Netto Junior, J; Padovani, CR. Lesões desportivas no atletismo: comparação entre informações obtidas em prontuários e inquéritos de morbidade referida. Rev Bras Med Esporte 2004; 10(1): 1-8.

102- Okura, Y; Urban, LH; Mahoney, DH; Jacobsen, SJ; Rodeheffer, RJ. Agreement between self-report questionnaires and medical record data was substantial for diabetes, hypertension, myocardial infarction and stroke but not for heart failure. J Clin Epidemiol 2004; 57(10): 1096-1103.

103- Wu, SC; Li, CY; Ke, DS. The agreement between self-reporting and clinical diagnosis for selected medical conditions among the elderly in Taiwan. Public Health 1999; 114(2): 137-142.

104- Bergmann, MM; Jacobs, EJ; Hoffmann K; Boeing, H. Agreement of self-reported medical history: comparison of an in-person interview with a self-administred questionnaire. European Journal of Epidemiology 2004; 19(5): 411-416.

105- Jones, CJ; Rikli, RE; Beam, WC. A 30-s chair-stand test as a measure of lower body strength in community-residing older adults. Res Q Exerc Sport. 1999; 70(1):113-19.

106- CLASSIFICAÇÃO ESTATÍSTICA INTERNACIONAL DE DOENÇAS E PROBLEMAS RELACIONADOS À SAÚDE. Décima revisão, 2008. Disponível em: <http://www.datasus.gov.br/cid10/v2008/cid10.htm>. Acesso em: 28 de abril de 2009.

107- Lohman, TG. Advances in body composition assessment. Current issues in exercise sciences series. Monograph No.3. Chapaign, IL: Human Kinetics, 1992. 
108- WORLD HEALTH ORGANIZATION. (1988). Measuring obesity-classification and description of anthropometric data. Report of a WHO Regional Office Consultation on the Epidemiology of Obesity. Copenhagen, Denmark: WHO Regional Office for Europe, Nutrition Unit. (Document EUR/ ICP/ NUT 125)

109- Stevens, J; Truesdale, KP; Katz, EG; Cai, J. Impact of Body Mass Index on Incident Hypertension and Diabetes in Chinese Asians, American Whites, and American Blacks. Am J Epidemiol 2008; 167(11):1365-1374.

110- Sebastião, E; Christofoletti, G; Gobbi, S; Hamanaka, AYY. Atividade física e doenças crônicas em idosos de Rio Claro-SP. Motriz 2008; 14(4): 381-388.

111- Olinto, MTA; Nacul, LC; Gigante, DP; Costa, JSD; Menezes, AMB; Macedo, S. Waist circumference as a determinant of hypertension and diabetes in Brazilian women: a population-based study Public Health Nutrition 2003; 7(5): 629-635.

112- Santos, A-C; Ebrahim, S; Barros, H. Alcohol intake, smoking, sleeping hours, physical activity and the metabolic syndrome. Preventive Medicine 2007; 44(4): 328-334.

113- Shankar, A; Klein, R; Klein, BEK. The association among smoking, heavy drinking, and chronic kidney disease. Am J Epidemiol 2006; 164(3): 263-271.

114- Abete, P; Ferrara, N; Cacciatore, F; Sagnelli, E; Manzi, M; Carnovale, V; et al. High Level of Physical Activity Preserves the Cardioprotective Effect of Preinfarction Angina in Elderly Patients. J Am Coll Cardiol 2001; 38(5):1357- 65.

115- Manini, TM; Everhart, JE; Patel, KV; Schoeller, DA; Colbert, LH; Visser, M; et al. Daily Activity Energy Expenditure and Mortality Among Older Adults. JAMA 2006; 296(2):171-179.

116- Longo, GZ; Das Neves, J; Luciano, VM; Peres, M. Prevalência de Níveis Pressóricos Elevados e Fatores Associados em Adultos de Lages/SC. Arq Bras Cardiol 2009; 93(4): 387-394.

117- Onat, A; Hergenc, TG; Keley, I; Dog, Y; Tqrkmen, S; Sansoy, V. Sex difference in development of diabetes and cardiovascular disease on the way from obesity and metabolic syndrome Prospective study of a cohort with normal glucose metabolism. Metabolism Clinical and Experimental 2005; 54(6): 800- 808.

118- Halldin, M; Rosell De Faire, MRU; Helle'Nius, ML. The metabolic syndrome: Prevalence and association to leisure-time and work-related physical activity in 60-year-old men and women. Nutrition, Metabolism \& Cardiovascular Diseases 2007; 17(5): 349 357. 
119- Heitmann, BL; Hills, AP; Frederiksen, P; Ward, LC. Obesity, Leanness, and Mortality: Effect Modification by Physical Activity in Men and Women. Obesity 2008; 17(1):136-142.

120- Neter, JE; Stam, BE; Kok, FJ; Grobbee, DE; Geleijnse, JM. Influence of Weight Reduction on Blood Pressure: A Meta-Analysis of Randomized Controlled Trials. Hypertension. 2003; 42(5): 878-884.

121- Kronenberg, F; Pereira, MA; Schmitz, MKH; Arnett, DK; Evenson, KR; Crapo, RO; et al. Influence of leisure time physical activity and television watching on atherosclerosis risk factors in the NHLBI Family Heart Study. Atherosclerosis 2000; 153(2): 433-443.

122- Poirier, P; Lemieux, I; Mauriège, P; Dewailly, E; Blanchet, C; Bergeron, J; Després, JP. Impact of Waist Circumference on the Relationship Between Blood Pressure and Insulin: The Quebec Health Survey. Hypertension 2005; 45(3): 363-367.

123- Franco, OH; LAET, C; PEETERS A; JONKER J; MACKENBACH, J; NUSSELDER W. Effects of Physical Activity on Life Expectancy With Cardiovascular Disease. Arch Intern Med. 2005; 165(20): 2355-2360.

124- Hu, G; Tuomilehto, J; Silventoinen, K; Barengo, NC; Peltonen, M; Jousilahti, P. The effect of physical activity and Body Mass Index on cardiovascular, cancer and all cause mortality among 47212 middle-aged finnish men and women. Int J Obesity 2005; 29: 894-902.

125- Matthews, CE; Jurj, AL; Shu, X; Li, HL; Yang, G; Li, Q; Gao, YT; Zheng, W. Influence of Exercise, Walking, Cycling, and Overall Nonexercise Physical Activity on Mortality in Chinese Women. Am J Epidemiol 2007; 165(12):1343-1350.

126- Feskanich, D; Willett, W; Colditz, G. Walking and Leisure-Time Activity and Risk of Hip Fracture in Postmenopausal Women. JAMA 2002; 288(18):2300-2306. 
ANEXO 1 - ANAMNESE

\section{IDENTIFICAÇÃO}

Prontuário $\mathrm{N}^{\circ}$ :

Data:

Nome:

Sexo: feminino ( ) masculino ( )

nasc.:

Etnia: branca ( ) negra ( ) parda ( ) amarela ( ) indígena

Estado civil: solteiro ( ) casado ( ) divorciado ( ) viúvo ( )

CONDIÇÃO SÓCIO ECONÔMICA - ( 0 - 1 - 2 - 3 - 4 - 5 - 6 )

Automóvel

Televisor

Empregada doméstica

Rádio

Máquina de lavar roupa

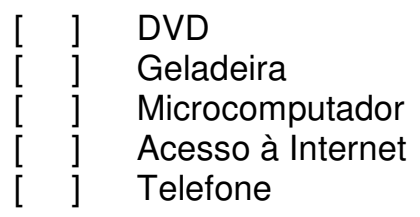

Dias/ semana:

\section{ESCOLARIDADE}

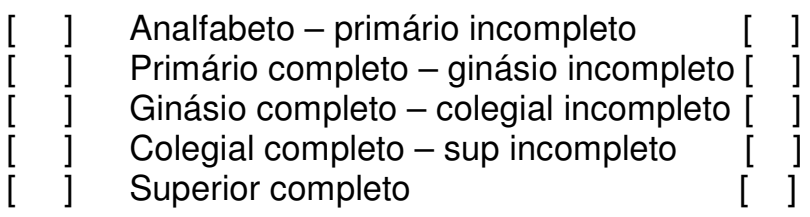

ATIVIDADE FÍSICA:

Tipo:

Observações:

\section{ANAMNESE DE SAÚDE}

PA: $\mathrm{mmHg}$

Tempo de doença:

FATORES DE RISCO

Fumante: $\quad \operatorname{sim}($ ) não ( )

Hábito alcoólico: $\operatorname{sim}($ ) não ( )

ANTROPOMETRIA

peso:_ $\mathrm{Kg} \quad$ estatura:__ $\mathrm{cm}$

Circunferências cintura: $\mathrm{cm}$

OBSERVAÇÕES: 
ANEXO 2 - INQUÉRITO DE MORBIDADES

\begin{tabular}{|c|c|c|c|c|}
\hline Doença & $\begin{array}{c}\text { Tempo } \\
\text { diagnóstico }\end{array}$ & $\begin{array}{l}\text { Ainda está } \\
\text { doente? }\end{array}$ & $\begin{array}{l}\text { Medicação } \\
\text { atual }\end{array}$ & OBSERVAÇÕES \\
\hline Colesterol alto & & $s() n()$ & $S() n()$ & \\
\hline Diabetes tipo II & $\ldots$ anos & $s() n()$ & $\mathrm{s}(\mathrm{)}) \mathrm{n}()$ & \\
\hline Hipertiroidismo & $\ldots$ anos & $\mathrm{s}(\mathrm{)}) \mathrm{n}(\mathrm{)}$ & $S() n()$ & \\
\hline Hipotiroidismo & __anos & $\mathrm{s}(\mathrm{)}) \mathrm{n}(\mathrm{)}$ & $S() n()$ & \\
\hline Arritmia & __ anos & $\mathrm{s}(\mathrm{)}) \mathrm{n}(\mathrm{)}$ & $S() n()$ & \\
\hline Infarto & $\ldots$ anos & $s() n()$ & $S() n()$ & \\
\hline Angina & __ anos & $\mathrm{s}(\mathrm{)}) \mathrm{n}(\mathrm{)}$ & $S() n()$ & \\
\hline Câncer & $\ldots$ anos & $\mathrm{s}(\mathrm{)}) \mathrm{n}(\mathrm{)}$ & $\mathrm{S}(\mathrm{)}) \mathrm{n}(\mathrm{)}$ & \\
\hline Osteoporose & $\ldots$ anos & $\mathrm{s}(\mathrm{)}) \mathrm{n}(\mathrm{)}$ & $S() n()$ & \\
\hline Artrite/ Artrose & _ anos & $\mathrm{s}(\mathrm{)}) \mathrm{n}(\mathrm{)}$ & $S() n()$ & \\
\hline Hérnia de Disco & $\ldots$ anos & $s() n()$ & $S() n()$ & \\
\hline Lombalgias & $\ldots$ anos & $\mathrm{s}(\mathrm{)}) \mathrm{n}(\mathrm{)}$ & $S() n()$ & \\
\hline \multirow[t]{4}{*}{ Escoliose } & $\ldots$ anos & $\mathrm{s}(\mathrm{)}) \mathrm{n}(\mathrm{)}$ & $S() n()$ & \\
\hline & __anos & $\mathrm{s}(\mathrm{)}) \mathrm{n}(\mathrm{)}$ & $S() n()$ & \\
\hline & $\ldots$ anos & $\mathrm{s}(\mathrm{)}) \mathrm{n}(\mathrm{)}$ & $S() n()$ & \\
\hline & __ anos & $s() n()$ & $S() n()$ & \\
\hline
\end{tabular}




\section{ANEXO 3 - NÍVEL DE ATIVIDADE FÍSICA}

\section{LEGENDA}

Nunca/ raramente/ algumas vezes/ freqüentemente/ sempre

$(1-2-3-4-5)$

Muito freqüentemente/ freqüentemente/ raramente/ algumas vezes/ nunca

$(5-4-3-2-1)$

\section{ATIVIDADES OCUPACIONAIS}

1. Qual sua principal ocupação?

2. sentado no trabalho

3. em pé no trabalho

4. andar no trabalho

5. carregar carga pesada no trabalho

6. se sentir cansado após o trabalho

7. suar durante o trabalho

8. em comparação com pessoas da mesma idade, meu trabalho é

muito pesado/ mais pesado/ tão pesado quanto/ mais leve/ muito leve
$1($ ) 3( ) 5( )

$1($ ) 2( ) 3( ) 4( ) 5( )

$1($ ) 2( ) 3( ) 4( ) 5( )

$1(\mathrm{)} 2(\mathrm{)}) 3(\mathrm{)} 4(\mathrm{)}) 5(\mathrm{)}$

1( ) 2( ) 3( ) 4( ) 5( )

$5($ ) 4( ) 3( ) 2( ) 1( )

$5($ ) $4($ ) $3($ ) $2($ ) $1($ )

5() 4() 3() 2() 1()

\section{ATIVIDADES DE LAZER E EXERCíCIO}

9. você pratica esportes?

tipo de esporte horas por semana

meses por ano

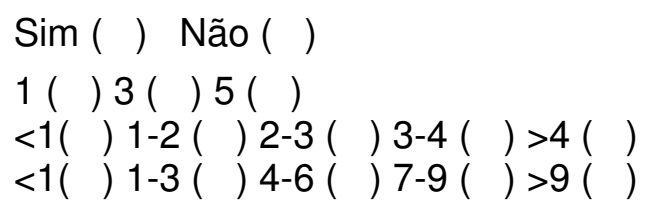

10. em comparação com pessoas da mesma idade, minha atividade durante o lazer é muito maior/ maior/ a mesma/ menor/ muito menor 5() 4() 3() 2() 1()

11. suor nas horas de lazer 5( )4( )3( )2( )1( )

12. prática de esportes no tempo livre 1( )2( )3( )4( )5( )

13. assistir televisão no tempo livre 1( )2( )3( )4( )5( )

14. caminhar durante tempo livre 1( )2( )3( )4( )5( )

15. andar de bicicleta no tempo livre 1( )2( )3( )4( )5( )

16. quantos minutos você caminha ou anda de bicicleta por dia, para o trabalho, escola, etc $(<5 / 5-15 / 15-30 / 30-45 />45)$ 
